\title{
An experimental and CFD modeling study of suspended droplets evaporation in buoyancy driven convection
}

\author{
A.E. Saufi ${ }^{\mathrm{a}, *}$, R. Calabria ${ }^{\mathrm{b}}$, F. Chiariello ${ }^{\mathrm{b}}$, A. Frassoldati ${ }^{\mathrm{a}}$, A. Cuoci ${ }^{\mathrm{a}}$, T. Faravelli $^{\mathrm{a}}$, P. Massoli $^{\mathrm{b}}$ \\ ${ }^{a}$ Department of Chemistry, Materials, and Chemical Engineering G. Natta, P.zza Leonardo da Vinci 32, 20133 Milano, Italy \\ ${ }^{\mathrm{b}}$ Istituto Motori - CNR, Naples, Italy
}

\section{H I G H L I G H T S}

- An analysis on the evaporation of acetic acid and ethylene glycol is presented.

- A multiregion approach for the fluidsolid heat transfer is adopted.

- The fiber transports heat towards the liquid, which distribute it by convection.

- The agreement with the experiments is excellent for all the cases.

- Internal convection influences the preferential vaporization in the mixture.

\section{A R T I C L E I N F O}

\section{Keywords:}

Droplet

Evaporation

DropletSMOKE

Fiber

Thermocouple

Convection
G R A P H I C A L A B S T R A C T

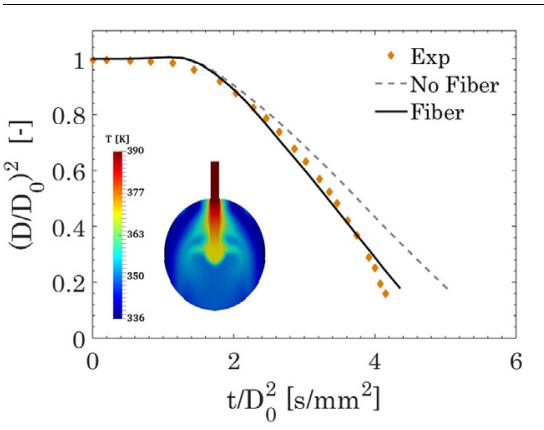

\begin{abstract}
A B S T R A C T
A detailed analysis on the evaporation of acetic acid and ethylene glycol droplets is performed experimentally and numerically. The isolated droplet is positioned in a combustion chamber, suspended on a thermocouple and evaporated in buoyancy driven convection, following the thermal history throughout the droplet lifetime. The experiments provide quantitative and qualitative data on the evaporation physics of acetic acid, ethylene glycol and their mixture. The data are then modeled adopting the multiphase CFD code DropletSMOKE ++ , describing the flow field around the droplet, the heating rate and the evaporation process. The main novelty introduced in this work is a multiregion approach to describe the solid fiber, which allows to model the conjugate heat transfer with the liquid and the gas phase, as well as its impact on the droplet evaporation. DropletSMOKE ++ results show a good agreement with the experimental data, regarding both the diameter decay and the liquid temperature, whose internal distribution in the liquid is shown to be highly affected by the heat flux from the fiber (which can contribute up to $30-40 \%$ in the total heat flux on the droplet). The effect of the thermocouple on the evaporation rate has been highlighted simulating the same experiments considering the solid as adiabatic, showing in this case a large underprediction of the vaporization rate and confirming the need of a detailed model for the fiber to correctly predict the vaporization phenomenon. The mixture evaporation has been investigated, emphasizing the importance of adopting a detailed thermodynamic model (which includes activity coefficients) and the impact of the mixture non-ideality on the evaporation process. The mixture also exhibits preferential vaporization, facilitated by the internal convection in the liquid phase.
\end{abstract}

\footnotetext{
* Corresponding author.

E-mail address: abdessamade.saufi@polimi.it (A.E. Saufi).
} 


\section{Nomenclature \\ Acronyms \\ ASOG Analytical Solution Of Groups \\ $\mathrm{CH} 2 \mathrm{OHCH} 2 \mathrm{OH}$ Ethylene Glycol \\ $\mathrm{CH} 3 \mathrm{CO} 2 \mathrm{H}$ Acetic Acid \\ FPBO Fast Pyrolysis Bio Oil \\ UNIFAC UNIversal Functional-group Activity Coefficients \\ $V L E \quad$ Vapor-Liquid Equilibrium \\ VOF Volume Of Fluid \\ Greek letters

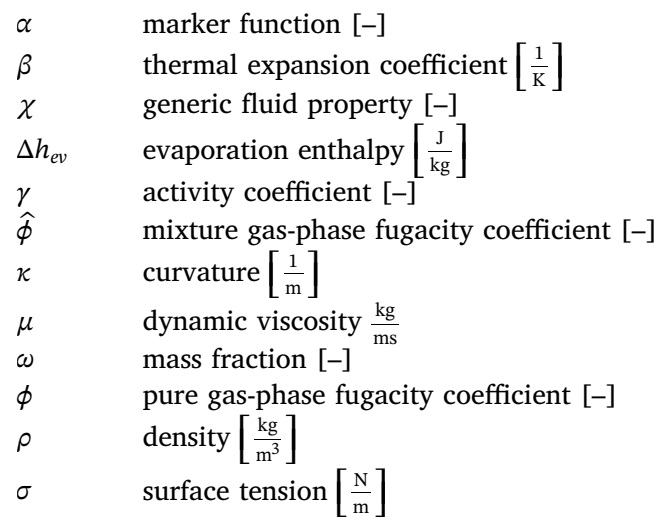

Roman letters

$\begin{array}{ll}\dot{m} & \text { evaporative flux }\left[\frac{\mathrm{kg}}{\mathrm{m}^{3} \mathrm{~s}}\right] \\ \dot{Q} & \text { heat flux }[\mathrm{W}] \\ \mathscr{D} & \text { mass diffusion coefficient }\left[\frac{\mathrm{m}^{2}}{\mathrm{~s}}\right]\end{array}$

\section{Introduction}

The study of the evaporation and combustion of isolated fuel droplets is currently an active area of research, mainly directed toward the better understanding and design of many engineering devices such as diesel engines and industrial burners. The CFD modeling of sprays relies on local source terms to describe the droplet heating, evaporation and combustion in a convective flow, usually based on semi-empirical correlations depending on dimensionless numbers (Re, Pr, Sc) $[1,2]$. Detailed results based on the direct numerical resolution of the differential equations (DNS) can help to build or improve reliable sub-grid scale models for the description of the vaporization rate, the internal mixing and the differential evaporation (for multicomponent mixtures) for a single droplet. In fact, the analysis is significantly simplified neglecting the complex droplet-droplet interactions (coalescence, breakup, etc.), both from an experimental $[3,4]$ and theoretical [5-7] point of view.

In the last years, many fuels have been investigated concerning isolated droplet vaporization with a particular attention toward alternative fuels such as pyrolysis-oil (POs) [8-10]. Pyrolysis oils (POs) are black-brownish liquids obtained by the condensation of vapors during the fast pyrolysis of vegetable biomass. In this context, a fundamental research activity is performed within the Residue2Heat project [11], which aims at developing a concept for renewable residential heating using FPBO (Fast Pyrolysis Bio Oil), to develop a proper surrogate mixture able to mimic the behavior of the real bio-oil. A nine-component mixture (water, acetic acid, ethylene glycol, glycol aldehyde, vanillin, HMW-lignin, levoglucosan, 2,5-dimethylfuran, oleic acid) has been defined and experimentally verified in comparison with the

\begin{tabular}{|c|c|}
\hline$\vec{f}$ & force $\left[\frac{\mathrm{N}}{\mathrm{m}^{3}}\right]$ \\
\hline$\vec{j}$ & mass flux $\left[\frac{\mathrm{kg}}{\mathrm{m}^{2} \mathrm{~s}}\right]$ \\
\hline$\vec{v}$ & velocity $\left[\frac{\mathrm{m}}{\mathrm{s}}\right]$ \\
\hline$C_{p}$ & constant pressure specific heat $\left[\frac{\mathrm{J}}{\mathrm{kg} \mathrm{K}}\right]$ \\
\hline$D$ & diameter $[\mathrm{m}]$ \\
\hline$H$ & height of the mesh $[\mathrm{m}]$ \\
\hline$k$ & thermal conductivity $\left[\frac{\mathrm{W}}{\mathrm{mK}}\right]$ \\
\hline$M_{w}$ & molecular weight $\left[\frac{\mathrm{kg}}{\mathrm{mol}}\right]$ \\
\hline Ns & Number of species $[-]$ \\
\hline$p$ & pressure $[\mathrm{Pa}]$ \\
\hline$p^{0}$ & vapor pressure $[\mathrm{Pa}]$ \\
\hline$r$ & radius $[\mathrm{m}]$ \\
\hline$t$ & time $[\mathrm{s}]$ \\
\hline$T$ & temperature $[\mathrm{K}]$ \\
\hline$v$ & mole volume $\left[\frac{\mathrm{m}^{3}}{\mathrm{~mol}}\right]$ \\
\hline$W$ & base radius of the mesh $[\mathrm{m}]$ \\
\hline$x$ & liquid phase mole fraction [-] \\
\hline$y$ & gas phase mole fraction [-] \\
\hline Subs & \\
\hline 0 & initial, reference \\
\hline$a m b$ & ambient, external \\
\hline $\operatorname{conv}$ & convective \\
\hline$d$ & diffusive \\
\hline$e v$ & evaporation \\
\hline$f i b$ & fiber \\
\hline$G$ & gas \\
\hline$i$ & species $i$ \\
\hline$L$ & liquid \\
\hline$s$ & solid \\
\hline
\end{tabular}

corresponding FPBO in single droplet experiments [12,11]. The experimental analyses and kinetic model development [13] were performed hierarchically, starting form pure components, then moving to their mixtures and finally the complete surrogate.

This work aims at analyzing, both experimentally and numerically, two of the proposed components for the bio-oil surrogate: acetic acid and ethylene glycol, as well as their mixture. The complex physics of the fuel droplets, suspended on a thin thermocouple in order to follow their thermal history, were studied in a single droplet combustion chamber by means of high speed shadowgraphs [12] to investigate evaporation process in detail. The heating rate at the thermocouple junction is provided by a resistive electric coil placed below the droplet, which induces buoyancy and an upward convective hot flow toward the liquid phase. The heating rate is firstly measured without the droplet and it is subsequently applied to the evaporation of acetic acid, ethylene glycol and their mixture, providing as output data the squared diameter decay and the liquid temperature profile.

Afterwards, the multiphase CFD code DropletSMOKE++[14] has been adopted to model the experiments, in order to obtain detailed data regarding the evaporation physics of the droplet. The DropletSMOKE+ + code, based on the VOF methodology, has been developed at Politecnico di Milano and it is specifically conceived for the detailed analysis of the evaporation of multidimensional fuel droplets in a convective flow. It has been validated against a large set of experimental data, with different fuels and in a wide range of operating conditions. In this work, the code has been further extended including the detailed description of the support fiber (i.e. the thermocouple), providing the temperature field of the fiber and a better insight on the heat transfer between the fluid and the solid phase. The literature on the topic 
includes the experimental and theoretical works of Yang et al. $[15,16]$, mainly concerning the impact of the fiber diameter on the evaporation rate. More recently, these analyses have been conducted by Rehman et al. [17], experimentally analyzing different supporting fiber sizes and materials and their effect on the droplet lifetime. Han et al. [18], studied this effect on multicomponent droplets, providing a numerical analysis based on a simple 1D model for both the droplet and the solid fiber and a quasi-steady state assumption for the gas phase. More detailed approaches have been proposed by Shringi et al. [19], also studying the impact of capillary fluxes on the droplet cooling, while Ghata et al. [20] implemented a VOF methodology for the fluid description, limiting however the study to reduced gravity conditions. Finally, Strizhak et al. [21] recently suggested a heat transfer model for tethered droplets, relying however on the Effective Thermal Conductivity model [6] for the temperature gradient and the circulation inside the droplets.

Concerning the CFD modeling, the main novelty of this work is the introduction of a multiregion approach, widely known in the CFD community for conjugate heat transfer problems, combined with the VOF methodology. This is applied to study the heat transfer of a threephase system in which the solid fiber, the liquid droplet and the gaseous convective flow interact with each other. This is done without relying on any sub-model, semi-empirical correlation or approximate approach for the solid fiber or the droplet heating and vaporization, performing a complete detailed numerical simulation of the three phase system.

The paper organization includes a detailed description of the experimental device (combustion chamber) for the analysis of the droplet evaporation, followed by a brief presentation on the main features of the DropletSMOKE++ code, presented in detail in the reference work [14], and its numerical implementation. The experimental heating rate detected by the thermocouple is then reconstructed and applied to the evaporation of acetic acid, ethylene glycol and their mixture. The results are then presented and discussed, with a particular focus on the impact of the support fiber on the vaporization physics and on the liquid temperature distribution. An analysis on the mixture non-ideality is reported in order to stress the importance of a detailed thermodynamics to correctly predict the evaporation rate. Finally, the internal velocity field in the liquid phase is reported, highlighting its role in the preferential vaporization of the mixture.

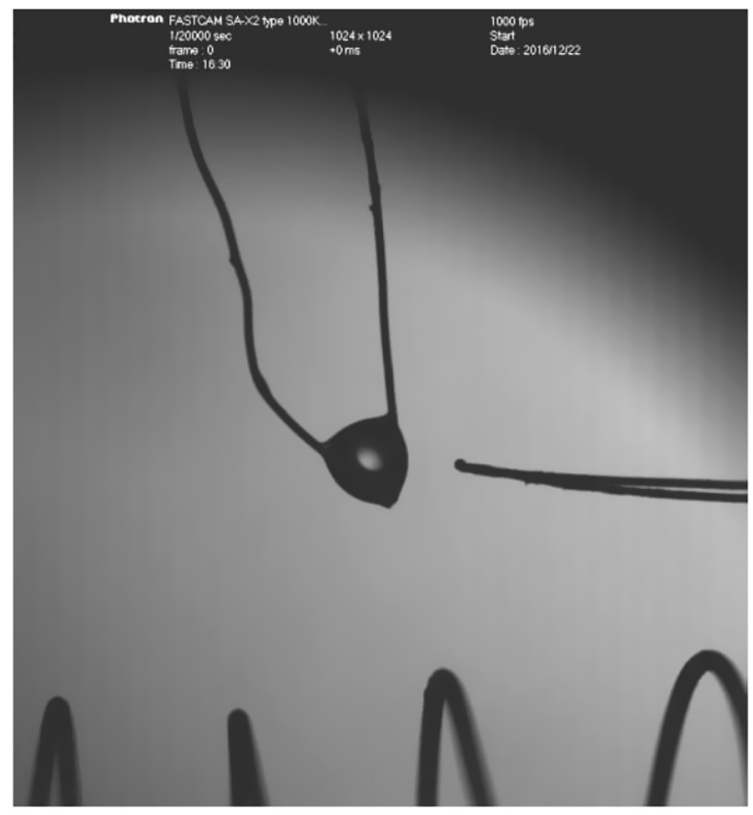

Fig. 2. Detail of the suspended droplet.

\section{Experimental configuration}

The evaporation studies on fuel droplets discussed in this work were carried out in a combustion cell designed to analyze the thermal behavior of single fuel droplets. The cell allows to realize the experiments in a wide range of operating conditions, controlling the heating rate (up to $1000 \mathrm{~K} / \mathrm{s}$ ), the pressure (from $0.1 \mathrm{kPa}$ to $10 \mathrm{MPa}$ ) and the atmosphere composition (usually nitrogen or air). The cell was originally realized in the framework of the European Project "COMBIO - A New Competitive Liquid Biofuel for Heating" [12], in order to study the combustion of pyrolysis oils and their emulsions up to a pressure of $10 \mathrm{MPa}$. From the geometrical point of view, the cell is a cylindrical chamber in stainless steel AISI 316 provided with four optical accesses for the realization of different experimental configurations and diagnostic. The experimental configuration is presented in Fig. 1.

At the center of the cell a thermocouple is placed. The liquid fuel droplet is suspended on the thermocouple junction by means of a

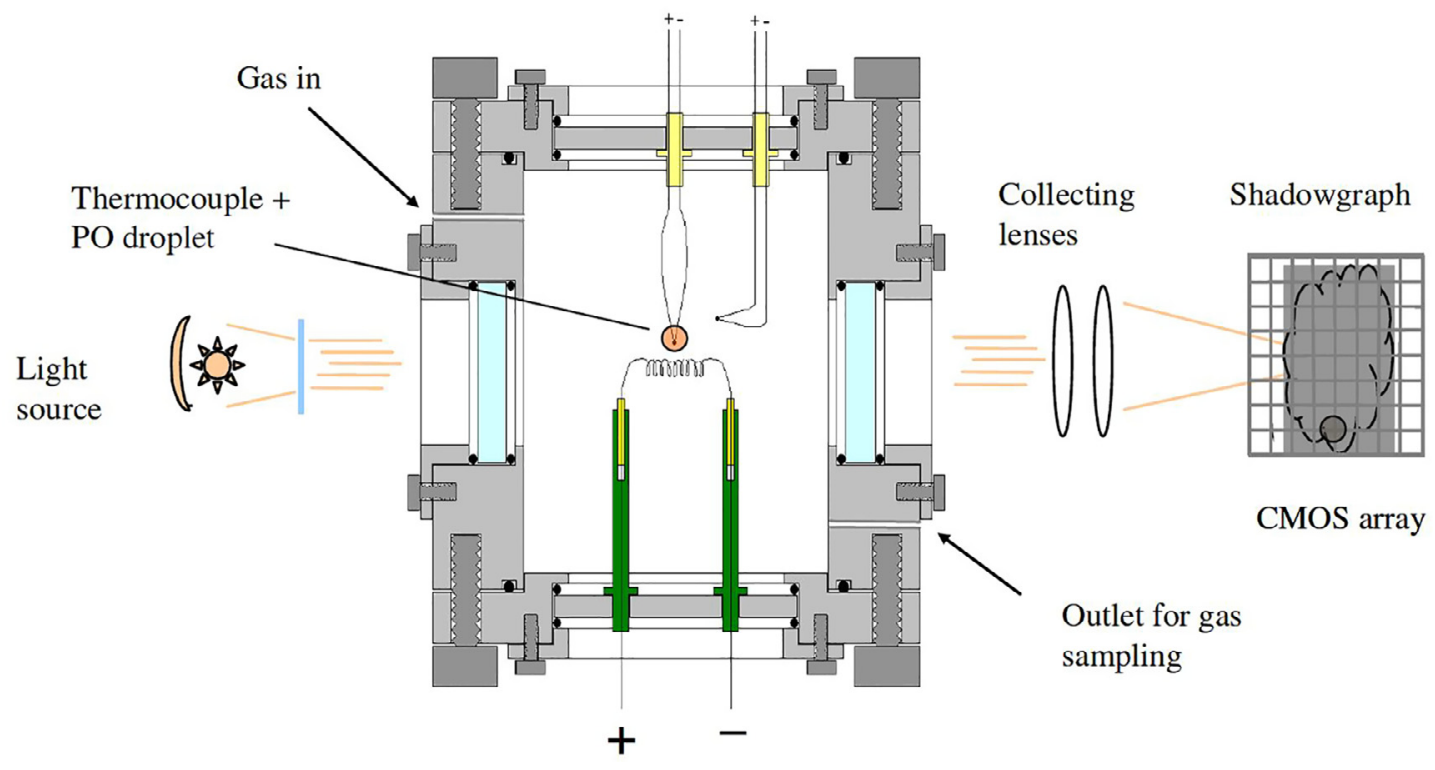

Fig. 1. Experimental configuration. 
microsyringe (Fig. 2). The heating of the fuel droplet is provided by an electric current passing through a coil of resistive material placed below the thermocouple, which releases heat by Joule effect. The evaporation of the droplet is followed by shadowgraph technique at high speed. A second thermocouple, placed laterally to the central one, is used to monitor the temperature in the area surrounding the droplet, which is particularly important for ignition experiments.

The thermocouples are of type $\mathrm{K}$ (chromel/allumel), with exposed junction, characterized by a good linearity of response in the range of $20{ }^{\circ} \mathrm{C}-1200^{\circ} \mathrm{C}$. In order to minimize intrusive effects of the thermocouple on the droplet thermal history, a wire diameter of $75 \mu \mathrm{m}$ has been adopted, while the junction diameter was limited to $125 \mu \mathrm{m}$. This choice has ensured a good adhesion of the droplet on the thermocouple junction even at high temperatures, when the surface tension and the viscosity of the fuel are considerably reduced. The signals of the thermocouples are acquired by means of the 4-channels transient recorder LeCroy Waverunner 104MXI-A. The image acquisition system is constituted by a CMOS high-speed (Photron Fastcam SA-X2) with a resolution of $1024 \times 1024$ pixels, 12-bit. The imaging is realized in shadowgraph configuration, in which the droplet is placed between a light source and the camera, with all these three elements perfectly aligned.

Movies obtained by the CMOS camera are saved as a sequence of individual images and analyzed with specific software for the extraction of relevant information. Through the analysis of the images of droplets in evaporation phase it is possible to extract quantitative information concerning the phenomenology, such as the equivalent diameter of the droplet over the time. By the coupled analysis of the output images and the signals from the thermocouple, the thermal history and the whole evaporation process of the droplet can be fully reconstructed.

\section{Mathematical model}

\subsection{Basic equations}

In the VOF methodology [22,23] a scalar marker function $\alpha$ is used to represent the liquid volumetric fraction of a two phase fluid, considering it as a single fluid with step-function properties. The $\alpha$ function varies from value 0 in the gas-phase to value 1 in the liquid phase and it is governed by the following transport equation:

$\frac{\partial \alpha}{\partial t}+\nabla \cdot(\vec{v} \alpha)=-\frac{\dot{m}}{\rho}-\frac{\alpha}{\rho} \frac{D \rho}{D t}$

The source term $\dot{m}$ is the evaporation flux which changes the liquid volume, while the second term accounts for the liquid density variation. The interface tracking is coupled with a single Navier-Stokes equation, solved for both phases:

$\frac{\partial(\rho \vec{v})}{\partial t}+\nabla \cdot(\rho \vec{v} \vec{v})=\nabla \cdot \mu\left(\nabla \vec{v}+\nabla \vec{v}^{T}\right)-\nabla p+\rho \vec{g}$

which provides the velocity fields. Finally, the energy equation is solved:

$\rho C_{p}\left(\frac{\partial T}{\partial t}+\vec{v} \nabla T\right)=\nabla \cdot(k \nabla T)+\beta \frac{D p}{D t}-\sum_{i=0}^{N s} \vec{j}_{d, i} C_{p, i} \nabla T-\sum_{i=0}^{N s_{L}} \dot{m}_{i} \Delta h_{e v, i}$

in which the interface cooling and the diffusion fluxes enthalpy of the species are included.

\subsection{Thermodynamics and multicomponent evaporation model}

The evaporation flux $\dot{m}_{i}$ is evaluated directly from the diffusion and convective fluxes at the gaseous interface, without the need of any particular evaporation model based on dimensionless numbers or empirical correlations [24]. We evaluate the surface mole fraction at the gas side, assuming thermodynamic equilibrium at the interface [25]:

$y_{i} l_{i n t}=\left.\frac{p_{i}^{0}(T) \phi_{i}\left(T, p_{i}^{0}\right) e^{\int_{p_{i}^{0}}^{p} \frac{v_{L}, i}{R T} d p} \gamma_{i}\left(T, p, x_{i}\right)}{p \widehat{\phi}_{i}\left(T, p, y_{i}\right)} x_{i}\right|_{\text {int }}$

where $p_{i}^{0}(T)$ is the vapor pressure of species $i, \phi_{i}$ is the gas-phase fugacity coefficient for the pure species and $\hat{\phi}_{i}$ is the gas-phase mixture fugacity coefficient. The exponential term represents the Poynting correction, while $x_{i} l_{\text {int }}$ and $y_{i} l_{\text {int }}$ are the liquid and gas mole fractions of species $i$. Finally, $\gamma_{i}$ is the activity coefficient for non-ideal mixtures, evaluated with the UNIFAC approach [26]. The conversion to mass fraction is straightforward:

$\left.\omega_{i}^{G}\right|_{\text {int }}=\frac{M_{w, i}}{M_{w}} y_{i} l_{\text {int }}$

This interfacial mass fraction $\left.\omega_{i}^{G}\right|_{\text {int }}$ is evaluated on the whole liquid phase and then advected and diffused towards the gas phase, simulating the vapor transport:

$\rho_{G}\left(\frac{\partial \omega_{i}^{G}}{\partial t}+\vec{v} \nabla \omega_{i}^{G}\right)=-\nabla \cdot \vec{j}_{d, i}^{G}$

where the diffusive flux $\vec{j}_{d, i}^{G}$ is computed as [14]:

$\vec{j}_{d, i}^{G}=-\rho^{G} \mathscr{D}_{i}^{G} \frac{M_{w, i}}{M_{w}} \nabla y_{i}$

The vapor presence in the gas phase can now be used to compute the evaporating flux $\dot{m}_{i}$ for each species, accounting for both the convective and the diffusive fluxes:

$\dot{m}_{i}=\left(\left.\rho \vec{v} \omega_{i}^{G}\right|_{\text {int }}-\rho \mathscr{D}_{i} \frac{M_{w, i}}{M_{w}} \nabla y_{i} l_{\text {int }}\right) \nabla \alpha$

where $|\nabla \alpha|$ accounts for the local surface area per unit volume, imposing the evaporation flux only at the interface. The vaporizing flux $\dot{m}_{i}$ is then included as a source term for the liquid species transport equation:

$\rho_{L}\left(\frac{\partial \omega_{i}^{L}}{\partial t}+\vec{v} \nabla \omega_{i}^{L}\right)=-\nabla \cdot \vec{j}_{d, i}^{L}-\dot{m}_{i}$

where the diffusive flux in the liquid phase is computed with a pseudoFick approach [27]:

$\vec{j}_{d, i}^{L}=-\rho^{L} \mathscr{D}_{i}^{L} \frac{M_{w, i}}{M_{w}} \nabla x_{i}$

The final mass fraction field of species $i$ is simply the superposition of the two fields:

$\omega_{i}=\alpha \omega_{i}^{L}+(1-\alpha) \omega_{i}^{G}$

\subsection{Surface tension modeling}

The main difficulty of the VOF approach is the modeling of surface tension driven flows. Very small droplets $(\sim 1-2 \mathrm{~mm}$ diameter) have a very large interface curvature $\kappa$, whose value is needed to compute the surface tension force $\vec{f}_{s}$ :

$\overrightarrow{f_{s}}=\sigma \kappa \nabla \alpha$

The numerical evaluation of curvature $\kappa$ from the $\alpha$ step-function is numerically challenging [28] and errors in this calculation lead to unphysical velocities around the interface called parasitic currents, which can easily propagate and eventually destroy the droplet. Many ways have been proposed to reduce this problem $[29,30]$, but none of them is able to completely eliminate spurious currents for the cases of our interest, concerning small evaporating droplets. In order to overcome this problem, a centripetal force $\vec{f}_{m}$ directed towards the droplet center has 
been imposed, in order to keep it strongly held to the thermocouple junction even when gravity is present. In this system surface tension force $\vec{f}_{s}$ is not needed anymore and it can be neglected, eliminating parasitic currents directly from their source. The Navier-Stokes equation becomes:

$\frac{\partial(\rho \vec{v})}{\partial t}+\nabla \cdot(\rho \vec{v} \vec{v})=\nabla \cdot \mu\left(\nabla \vec{v}+\nabla \vec{v}^{T}\right)-\nabla p+\rho \vec{g}+\vec{f}_{m}$

This by-passing technique has been proposed by Saufi et al. [14] and represents one of the strengths of our numerical code, since it allows to model small droplets dynamics without worrying about spurious currents. Surface tension is suppressed, eliminating the parasitic flows from their very source and allowing to model the whole evaporation process, whatever the droplet size. More details and analyses about this technique are presented in the specific work on the DropletSMOKE++ code [14].

\subsection{Multiregion approach for conjugate heat transfer}

One of the main novelties of this paper is the implementation of a multiregion approach in order to account for the solid phase representing the thermocouple wire. The solid fiber rapidly conducts heat towards the droplet, representing an additional preferential path for the heat fluxes on the liquid phase, which can perturb the evaporation process $[31,15,32]$. In this work we implemented an extension of the DropletSMOKE++ code, in which we combine the VOF methodology to describe the two-phase fluid and a multiregion approach for the sole solid region with a very high level of detail. The heat transfer across the phases can be modeled accounting for the real geometry of the system, with no need of semi-empirical correlations or approximate approaches to account for the fiber. The fluid and the solid regions are independently meshed, solved and connected by means of dynamic boundary conditions, providing a full detailed numerical simulation of the three-phase system. In particular, a simple heat conduction equation is solved for the solid phase:

$\rho_{s} C_{p, s} \frac{\partial T_{s}}{\partial t}=\nabla \cdot\left(k_{s} \nabla T_{s}\right)$

while the fluid temperature field is provided by Eq. (3). The external surface of the solid fiber is the contact boundary between the phases. The boundary conditions which apply describe the conservation of heat fluxes across the boundary as well as the continuity of the temperature field. Eqs. (3) and (14) are solved in a closed loop until convergence. The solid properties values are $k_{s}=29.7 \frac{\mathrm{W}}{\mathrm{mK}}, \rho_{s}=8600 \frac{\mathrm{kg}}{\mathrm{m}^{3}}$ and $C_{p, s}=523$ $\frac{\mathrm{J}}{\mathrm{kg} \mathrm{K}}$.

\subsection{Fluid properties}

The OpenSMOKE++ library [33] is used to compute the fluid properties. The gas physical properties $\left(\rho, \mu, C_{p}, k, \mathscr{D}_{i}\right)$ are based on the kinetic theory of gases, while liquid properties $\left(\rho, \mu, C_{p}, k, \mathscr{D}_{i}, \Delta h_{e v, i}\right)$ are evaluated based on the correlations available in the Yaws and Prausnitz $[34,35]$ databases and thermophysical definition of the FPBO mixture developed within the Residue2Heat project [11]. The mixture properties to be used in the governing equations can be then computed. For a generic property $\chi$ :

$\chi=\chi_{L} \alpha+\chi_{G}(1-\alpha)$

\section{Numerical methodology}

The DropletSMOKE++ code is based on the open-source framework OpenFOAM ${ }^{\circledast}$, which allows to numerically solve the transport equations on complex geometries. The pressure-velocity coupling is implemented through the PIMPLE algorithm [36], while for the time discretization an

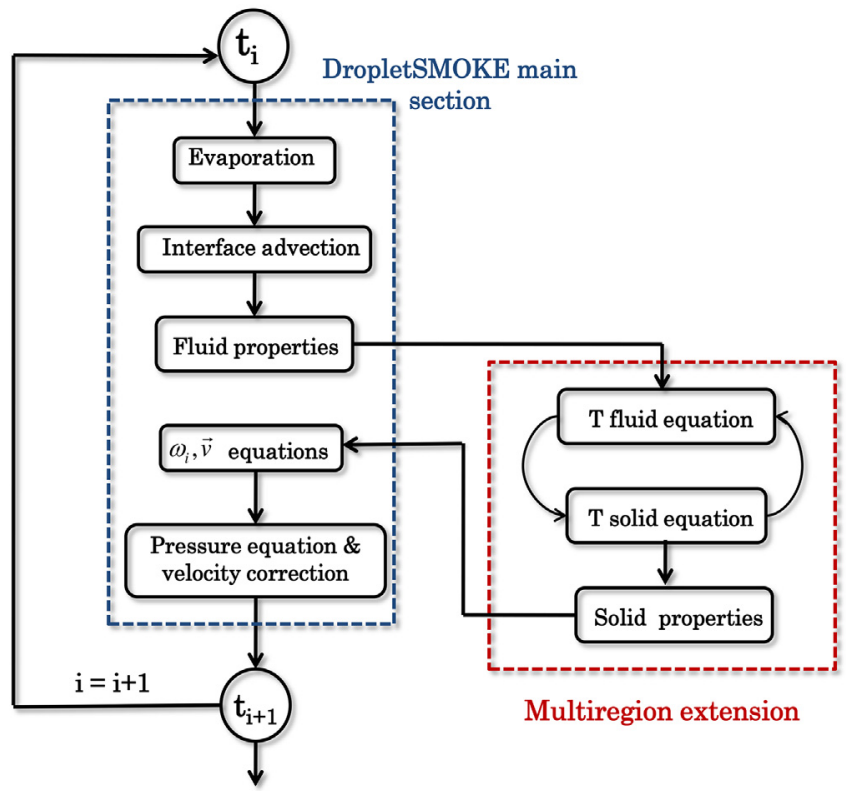

Fig. 3. Numerical steps of the DropletSMOKE++ code, including the multiregion extension for the description of the solid fiber.

implicit Euler method is adopted. A threshold Courant number (Co < 1) governs the time-step value. Gauss linear upwind scheme is used for spatial discretization of convective terms, while an orthogonal correction is adopted for Laplacian terms. The structure of the code is reported in Fig. 3, highlighting the multiregion extension.

\subsection{An alternative configuration for the CFD modeling}

The main geometrical dimensions of the experimental configuration (Fig. 1) are reported in Fig. 4(a). The CFD simulation of this domain would require a full 3D simulations, since the presence of the coil and the double-wire thermocouple does not leave any symmetry in the system to be exploited. A 3D simulation would be very difficult, not only concerning the domain construction, but mainly because of the high computational cost of the simulations. Therefore, in order to model the experimental device with a reasonable computational cost, an alternative geometrical configuration has been adopted, characterized by the presence of axial symmetry.

This alternative configuration is represented by the cylindric geometry in Fig. 4(b). The following differences with respect to the original one (Fig. 4 a) are introduced:

- Only a reduced portion of the whole domain is modeled, treating all the external boundaries of the new configuration as open (Fig. 4 b). The droplet is positioned far enough from the chamber boundaries to consider the wall perturbation on the evaporation process negligible. This allows to significantly reduce the number of computational cells.

- The thermocouple is modeled as a single vertical solid wire, approximate to a very thin cylinder, which ends with a small sphere representing the thermocouple junction. This creates an axial symmetry in the geometry which can be used to simplify the simulations.

- The original heating coil geometry is a horizontal metallic spring. In the alternative configuration in Fig. 4(b) it has been replaced with a planar single wire coil, which can be described within an axisymmetric geometry. The distance between the droplet and this planar coil in the new configuration is equal to the distance between the droplet and the axis of the horizontal spring in the real system (Fig. 4 a). This is done to preserve the "average" distance of the 


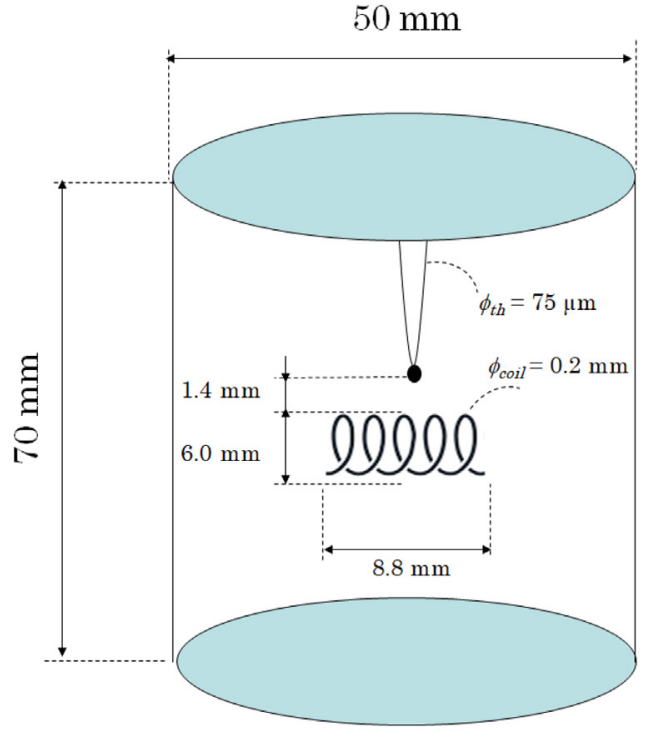

(a)
$10 \mathrm{~mm}$

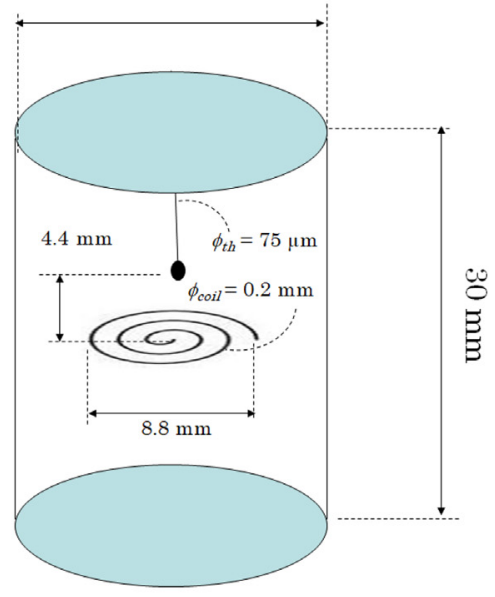

(b)

Fig. 4. Detailed geometrical dimensions of the original experimental configuration in (a). Alternative configuration used for the CFD simulations in (b).

heating system from the droplet, maintaining a realistic description of the experimental device.

\subsubsection{Computational mesh}

The computational mesh used for all the numerical cases proposed in this work has been built with the commercial CFD code AnsysFLUENT $^{\circledast}$ v19.2 and subsequently imported in OpenFOAM ${ }^{\circledR}$. The mesh is reported in Fig. 5. The geometry is $2 \mathrm{D}$ and axisymmetric, representing a slice of a cylinder having a radius $W=5 \mathrm{~mm}$ and a height $H=30 \mathrm{~mm}$. The fluid region (blue color) and the solid region (orange color) are independently meshed and then connected. The solid region represents the metallic fiber, a vertical cylindric wire $\left(r_{\text {wire }}=37.5 \mu \mathrm{m}\right)$ ending with a small supporting sphere $\left(d_{\text {fiber }}=125 \mu \mathrm{m}\right)$, around which the liquid droplet will be suspended. The front view of the planar heating coil in the new configuration (Fig. 4 b) is a series of circular holes (Fig. 5), having a diameter $d_{\text {coil }}=0.2 \mathrm{~mm}$. The number of cells is $\sim 60,000$, chosen by a sensitivity analysis on the cell size, with a maximum mesh non-orthogonality equal to 59.4 and a maximum Skewness of 1.4. A particularly fine mesh is imposed in the droplet region and around the heating coil.

\subsection{Reconstruction of the heating rate}

The metallic coil heats the surrounding atmosphere, moving the gas toward the droplet and triggering the evaporation process. However, the upward velocity of the hot gas and its temperature are generally not known, since they depend on the coil geometry, the current intensity and the material resistivity, which are usually not known with a sufficient accuracy. To overcome this problem, a preliminary experiment is performed in order to obtain the heating rate of the system:

- The droplet is taken out of the chamber. The thermocouple is now

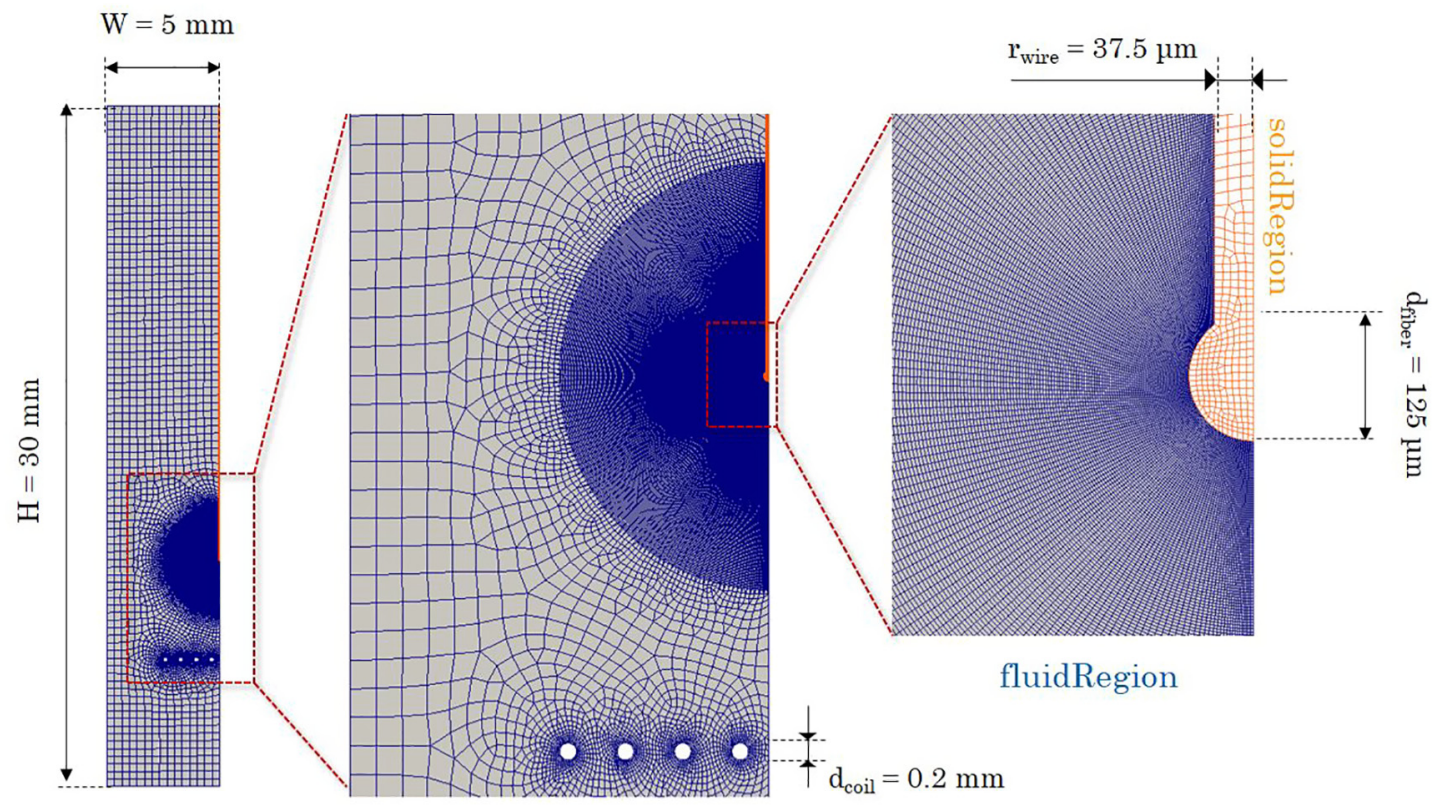

Fig. 5. Multiregion computational mesh used for the CFD simulations at three increasing levels of detail, from left to right. The fluid region mesh is reported in blue color, the solid region mesh (fiber) is reported in orange. 
directly exposed to the gaseous phase.

- The coil is electrically heated, providing buoyancy and the upward convective flow

- The temperature at the thermocouple junction is measured, providing the temperature profile and the heating rate in that point.

An example of the experimental temperature profile is presented in Fig. 6(a). The temperature profile in a generic fixed point gives an indirect information on the fluid flow velocity $\vec{v}$ and the local temperature gradient $\nabla T$ :

$\frac{\partial T}{\partial t}=-\vec{v} \nabla T+\frac{k}{\rho C_{p}} \nabla^{2} T$

The strategy is to perform a trial and error procedure, in which the boundary condition at the heating coil surface (Fig. 5) is tuned in order to generate an upward flow which provides a temperature profile at the thermocouple equal to the experimental one (Fig. 6a). Once this is done, the droplet is suspended on the thermocouple and evaporated, maintaining the same boundary condition at the coil surface.

There are many alternatives for the heating coil surface boundary condition. The best we found is represented by a time-varying boundary condition, imposed by a function of this type:

$T(t)=T_{\infty}-\left(T_{\infty}-T_{a m b}\right) e^{-\xi t}$

which physically represents the heating dynamics of the coil, which reaches a steady-state temperature $T_{\infty}$ in an environment at $T_{a m b}=285 \mathrm{~K}$. The steady-state temperature of the coil $T_{\infty}$ and $\xi$ (the rate of approach to the steady state value $T_{\infty}$ ) are the tuning parameters of the problem. Referring to Fig. 6(b), we need to find the optimal values of the parameters $T_{\infty}, \xi$ of the coil surface boundary condition (Eq. (17), gray dashed line in Fig. $6 \mathrm{~b}$ ), that provide a heating rate at the thermocouple (black line in Fig. 6 b) which fits the experiment. In the particular case presented in Fig. 6 , the tuning values are $T_{\infty}=1670 \mathrm{~K}$, $\xi=0.6 \mathrm{~s}^{-1}$.

The CFD simulation of Fig. 6 is presented in Fig. 7, at three different times. In Figs. $7(\mathrm{a}, \mathrm{b}, \mathrm{c})$ the heating dynamics of the coil can be clearly seen, increasing the gas temperature over $1600 \mathrm{~K}$ in few seconds. The gas flow (Figs. 7d, e, f) moves upward because of the buoyancy and increases the fiber junction temperature $(\sim 900 \mathrm{~K})$, with the heating rate presented in Fig. 6(a).

\subsection{Boundary conditions}

The geometry has five boundaries, referring to Fig. 5:

- inlet, outlet: the upper and bottom circular surfaces of the cylinder

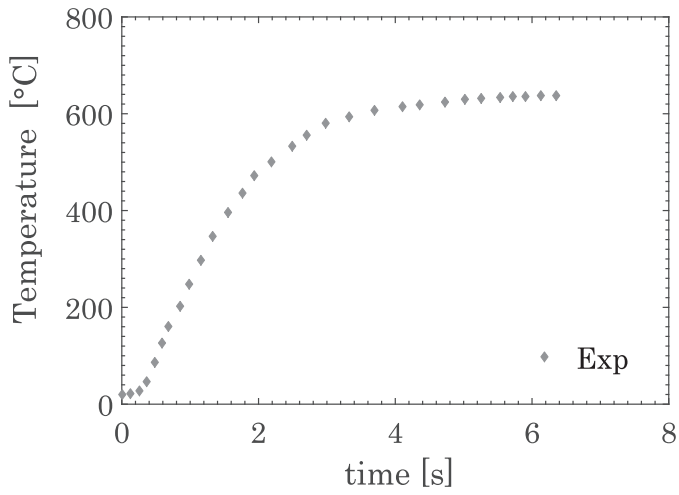

(a)
- outerWall: external lateral surface of the cylinder

- solidFiber: the external surface of the solid vertical fiber (thermocouple), in contact with the fluid region

- heatingCoil: the external surface of the heating coil

Since we are not modeling the whole volume (Fig. 4a), but only a portion of it (Fig. 4b) the first three boundaries are open, in which an inletoutlet condition apply (a zero gradient condition, that switches to a fixed value condition if the velocity vector next to the boundary points inside the domain, e.g. backward flow). The temperature condition at the solidFiber boundary describes the heat transfer with the external fluid region, whereas it represents a wall for all the other variables (velocity, species etc.). The coupled heat transfer is included in the turbulentTemperatureCoupledBaffleMixed boundary condition, available in OpenFOAM ${ }^{\circledast}$ for conjugate heat transfer problems [36]. The heatingCoil boundary presents a time dependent condition for temperature, which is needed to develop the correct heating rate at the thermocouple (Eq. (17)). The boundary conditions are summarized in Table 1.

\section{Description and modeling of the experimental cases}

The multiregion code DropletSMOKE++ is adopted to model the experimental data reported in Table 2. For each one of the cases an experimental heating rate at the thermocouple is provided and reconstructed (Table 3).

\subsection{Pure components evaporation}

The numerical simulation of Case 1 (Table 2) is presented in Fig. 8, where the acetic acid mass fraction (a, b, c), the temperature (d, e, f) and the velocity vector $(g, h, i)$ fields are reported at three different times. The heating coil generates an upward fluid flow starting the droplet evaporation process. The vapor distribution around the droplet $(\mathrm{a}, \mathrm{b}, \mathrm{c})$ is not uniform, due to the different temperature on the droplet surface. The hot gas heats the droplet mainly from below, causing a higher evaporation rate in the lower region (b). Moreover, the presence of the fiber contributes to increase the local surface temperature close to the solid (b, c) where the liquid is more exposed to the hot boundary, as reported by Strizhak et al. [21] for suspended water droplets. In particular, the temperature behavior of the fiber is reported in Figures (d, e, f). Outside the liquid phase, the solid is directly exposed to the external gas and the absorbed heat flux is directly transferred to the center of the droplet, where the temperature approaches a steady-state temperature $\left(\sim 100^{\circ} \mathrm{C}\right)$. The temperature field inside the fiber can be considered one dimensional, as already reported in previous works $[20,16,19]$.

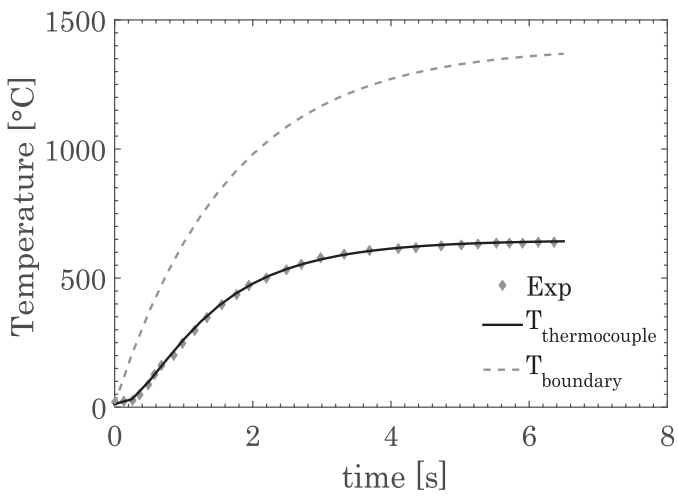

(b)

Fig. 6. Experimental temperature profile at the thermocouple junction (a). Matching of the CFD results with the experiment (Figure b, black line vs. points) and the relative boundary condition profile (gray dashed line) at the coil surface. 


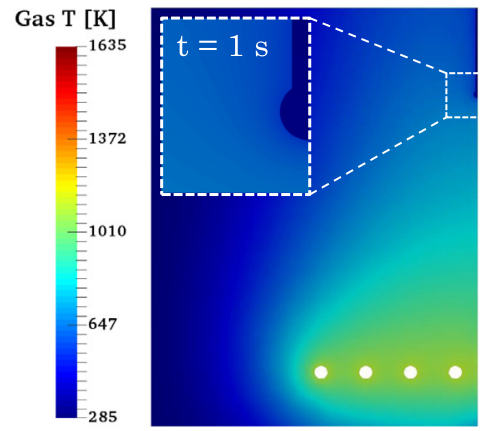

(a)

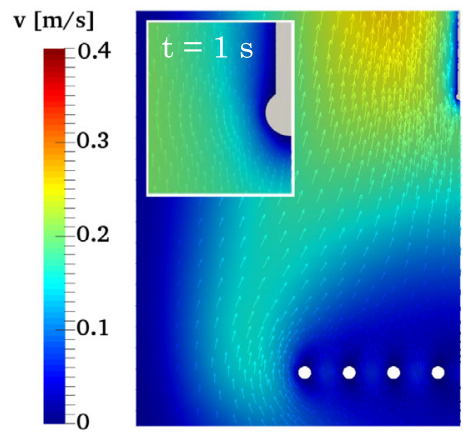

(d)

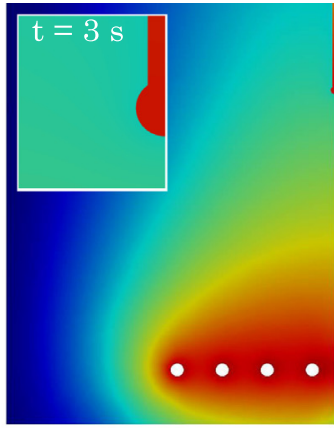

(b)

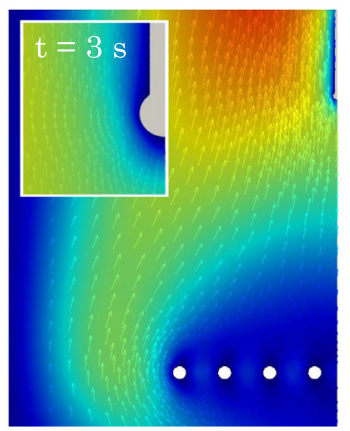

(e)
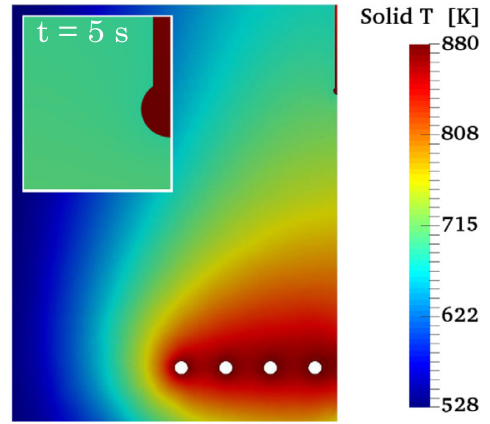

(c)

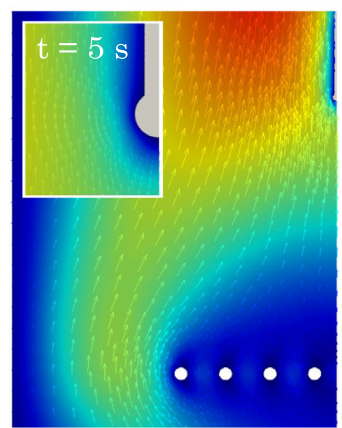

(f)

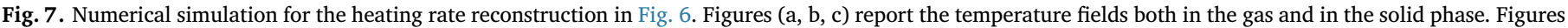
$(\mathrm{d}, \mathrm{e}, \mathrm{f})$ report the velocity vector field of the gas phase. Pictures taken at times $\mathrm{t}=1,2,5 \mathrm{~s}$. In each picture, a detail of the solid fiber is shown.

Table 1

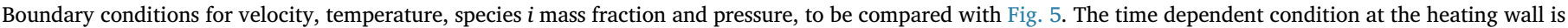
needed for the heating rate reconstruction.

\begin{tabular}{|c|c|c|c|c|}
\hline Boundary & $\vec{v}$ & Temperature & $\omega_{i}$ & $p$ \\
\hline inlet, outlet & inletOutlet & inletOutlet & inletOutlet & $p=p_{\text {ext }}$ \\
\hline outerWall & inletOutlet & inletOutlet & inletOutlet & $p=p_{\text {ext }}$ \\
\hline solidFiber & noSlip & fluidCoupling & $\nabla \omega_{i}=0$ & $\nabla p=0$ \\
\hline heatingCoil & noSlip & $T(t)=T_{\infty}-\left(T_{\infty}-T_{a m b}\right) e^{-\xi t}$ & $\nabla \omega_{i}=0$ & $\nabla p=0$ \\
\hline
\end{tabular}

Table 2

Experimental cases of evaporating droplets examined in this work.

\begin{tabular}{lllllll}
\hline Case & Species & $\begin{array}{l}D_{0} \\
{[\mathrm{~mm}]}\end{array}$ & $\begin{array}{l}T_{L}^{0} \\
{[\mathrm{~K}]}\end{array}$ & $\begin{array}{l}T_{G}^{0} \\
{[\mathrm{~K}]}\end{array}$ & $\begin{array}{l}\mathrm{p} \\
{[\mathrm{atm}]}\end{array}$ & Results \\
\hline 1 & Acetic Acid & 0.959 & 285 & 285 & 1 & Figs. 8 and 9 \\
2 & Ethylene Glycol & 0.940 & 285 & 285 & 1 & Fig. 10 \\
3 & Mixture & 0.928 & 285 & 285 & 1 & Figs. 11 and 14 \\
\hline
\end{tabular}

Table 3

Boundary condition at the heatingCoil boundary (Table 1, Fig. 5) for the experimental cases in Table 2.

\begin{tabular}{ll}
\hline Case & $T_{\text {boundary }}[\mathrm{K}]$ \\
\hline 1 & $T_{1}(t)=1670-(1670-285) e^{-0.6 t}$ \\
2 & $T_{2}(t)=1650-(1650-285) e^{-0.6 t}$ \\
3 & $T_{3}(t)=1670-(1670-285) e^{-0.65 t}$ \\
\hline
\end{tabular}

The plots in Fig. 9 report the normalized squared diameter decay of Case 1 (Figure a) and the liquid temperature profile measured by the thermocouple (Figure b). The temperature presents a sudden increase at the end of the experiment because the complete droplet evaporation exposes the thermocouple to the hot gas. The DropletSMOKE++ code can predict the experimental values with a good accuracy for both the diameter and the liquid temperature, including the final heating of the fiber. In order to better analyze the impact of the fiber, the same simulation has been performed considering the solidFiber boundary as adiabatic. The main effect is a significant reduction of the evaporation rate (Figure a) and the consequent increased lifetime of the droplet. Coherently, the liquid temperature is slightly lower when the solid heat transfer contribution is not accounted for (Figure b).

In Fig. 10 the results for Case 2 (Table 2) are presented, concerning ethylene glycol evaporation. The DropletSMOKE++ code is still able to predict the experimental values with reasonable accuracy, even if some deviation can be seen toward the end of the droplet lifetime (Figure a). The evaporation rate is underestimated in the latest stages of the experiment, leading to larger diameters and a slightly lower liquid temperature (Fig. 10 b). The same behavior, to a lesser extent, can be seen for acetic acid droplet simulations (Fig. 9).

This phenomenon is probably due to the formation of bubbles inside the liquid phase, which can be clearly seen experimentally. The solid fiber directly conducts heat into the liquid phase, increasing the internal liquid temperature and reaching a steady-state temperature $\left(\sim 160^{\circ} \mathrm{C}\right)$, not reached if the solid fiber is neglected (Fig. 10b). Moreover, the presence of the fiber surface facilitates the nucleation of bubbles on the solid surface, rapidly increasing the liquid consumption rate, the internal circulation and eventually the heat transfer on the droplet. The DropletSMOKE ++ code does not include a nucleation/ boiling sub-model, considering the phase transition as purely superficial 


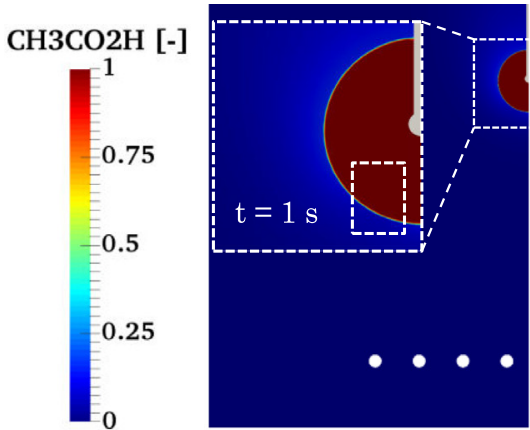

(a)

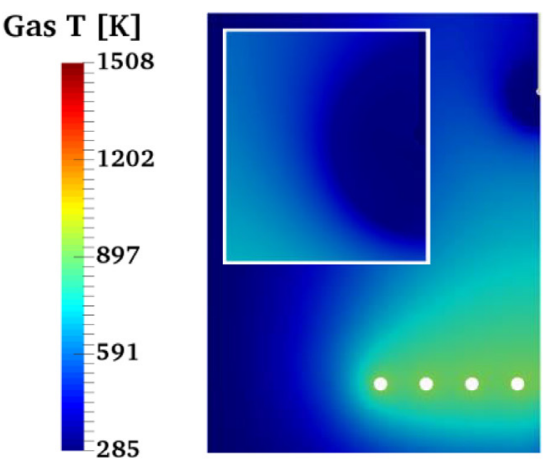

(d)

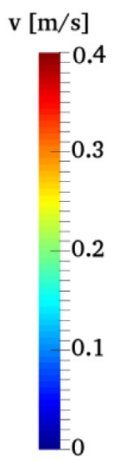

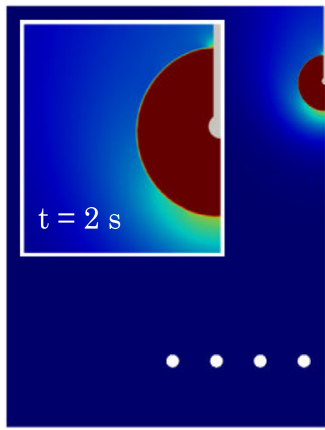

(b)
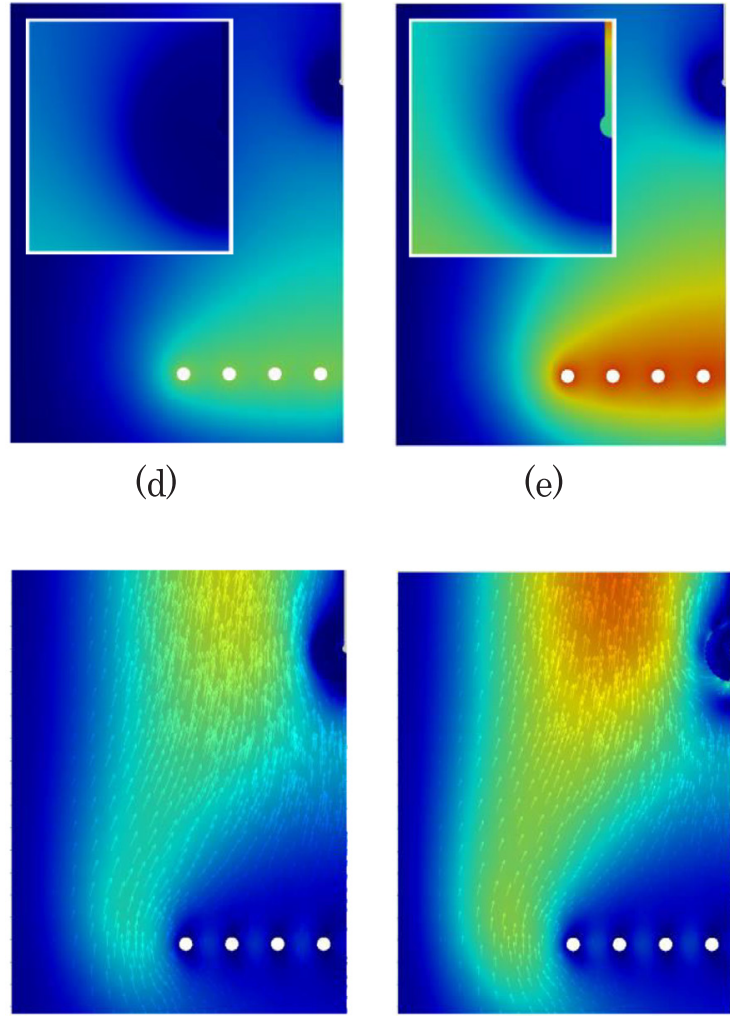

(g)

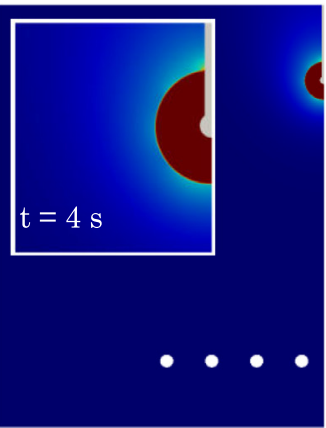

(c)

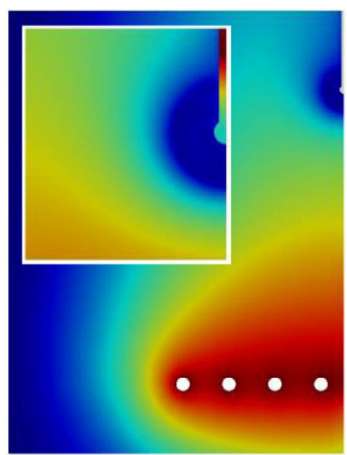

Solid T [K]

422

401

379

357

335 (e)

(f)

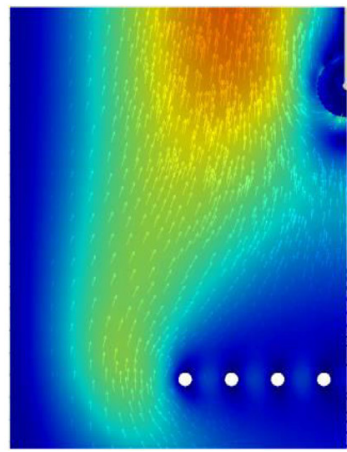

(h)

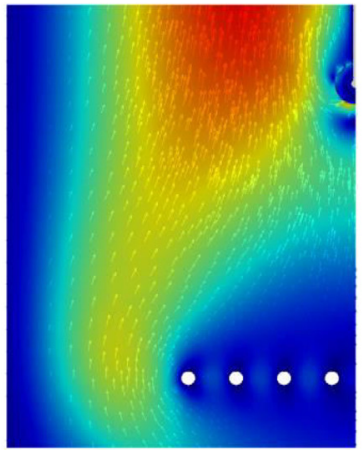

(i)

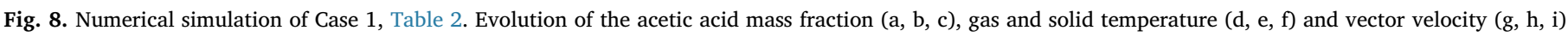
fields for times $\mathrm{t}=1 \mathrm{~s}, 2 \mathrm{~s}$ and $4 \mathrm{~s}$.

and not volumetric. This may cause an underestimation of the vaporization rate, leading to larger diameters toward the end of the droplet lifetime. As before, an additional simulation neglecting the solid heat transfer is presented, showing a significant underestimation of the vaporization rate as well as a lower steady state liquid temperature.

It is worth analyzing the temperature distribution in the liquid phase in order to emphasize the impact of the fiber. The temperature fields are reported in Figs. 9(c) for acetic acid (at time $t=3 \mathrm{~s}$ ) and Fig. 10(c) for ethylene glycol (at time $=5 \mathrm{~s}$ ), both for the liquid and the solid phase. The heat flux from the hot gas is conducted through the fiber, released in the liquid phase and distributed by the internal circulation. It is interesting to notice that the temperature distribution in the acetic acid droplet is more uniform if compared with ethylene glycol. This is due to the much higher viscosity of ethylene glycol, which reduces the internal velocities making the redistribution of the heat flux from the fiber more difficult. For both cases the maximum liquid temperature is found at the droplet center, where the liquid cannot evaporate. This creates favorable conditions for internal bubbles nucleation once the boiling temperature is reached (Figs. $9 \mathrm{~b}, 10 \mathrm{~b}$ ), indicating the reason for the slight delay of DropletSMOKE++ with respect to the experiments at the end of the evaporation.

\subsection{Mixture evaporation}

Case 3 in Table 2 regards the evaporation of a 1:1 (volumetric) mixture of acetic acid and ethylene glycol. In this case we have two species in the liquid phase, which are diffused and advected by the internal velocity field. Fig. 11 reports the evolution of acetic acid (a, b, c) and ethylene glycol (d, e, f) mass fraction. Ethylene glycol is the heavy species and it tends to concentrate in the droplet, while acetic acid evaporates faster. An internal mass fraction gradient is present (Figures b, e), which is rapidly homogenized by the strong internal convection.

Fig. 12 reports the normalized squared diameter decay of Case 3 


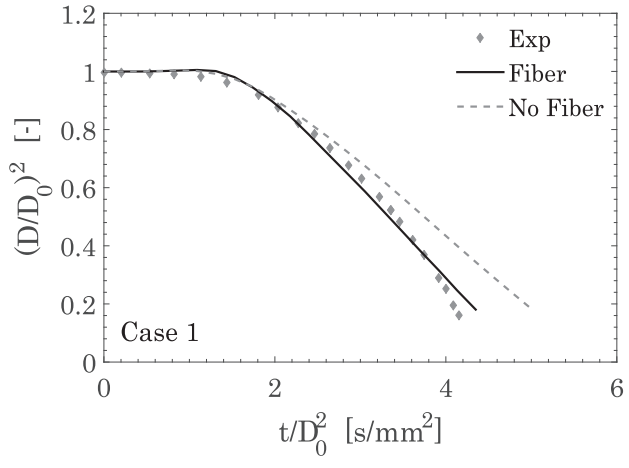

(a)

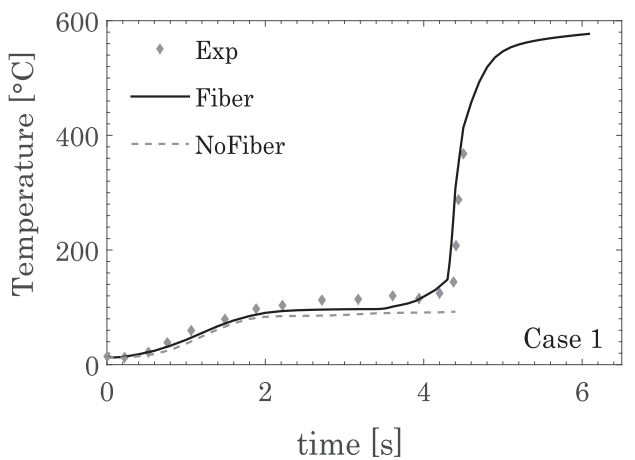

(b)

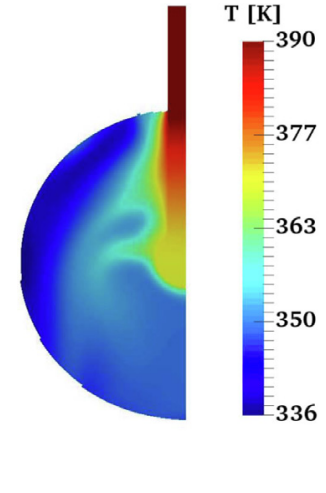

(c)

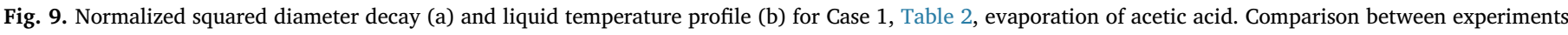

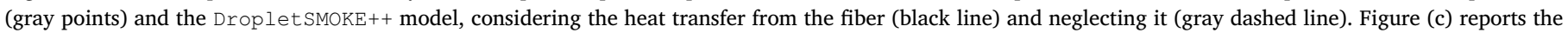
temperature distribution along the solid fiber and inside the liquid droplet at time $t=3 \mathrm{~s}$.

(Figure a) and the liquid temperature profile measured by the thermocouple (Figure b). While the liquid temperature is captured with good accuracy, the diameter decay shows a different trend. Comparing the model with the experiments, the evaporation is too fast at the initial stages and too slow towards the end of the vaporization process. Including the heat transfer with the fiber slightly improves the results (from the point of view of the droplet lifetime), but the qualitative trend does not change significantly. Moreover, since the final part of the evaporation is delayed, also the exposure of the thermocouple to the gas is shifted (Fig. 12 b). Since this discrepancy only appears for Case 3, it is worth investigating the mixture thermodynamics in detail.

\subsection{Wilson model for activity coefficients}

The vapor-liquid equilibrium (VLE) data of acetic acid-ethylene glycol mixture are taken from the experimental values from Schmid et al. [37] and reported in Fig. 13(a) (red points). The data are taken at $T=363 \mathrm{~K}$ and show a slightly negative deviation from the Raoult's law, suggesting the need of a non-ideal mixture assumption for the system (since the vapor phase can be safely considered ideal). In this work the activity coefficients $\gamma_{i}$ of the species were computed with the UNIFAC approach [26], considering the functional groups present in the molecules. However, as can be clearly seen in Fig. 13(a), the UNIFAC method does not show a significant deviation from the mixture ideality and therefore it is not able to correctly predict the experimental data for this specific mixture. The modified UNIFAC [38] and the ASOG
(Analytical Solution Of Groups) [39] methodologies predict a positive deviation of the VLE data from the ideality (not shown), leading to the fact that no predictive model for the activity coefficients can properly describe the acetic acid- ethylene glycol mixture. This may explain the discrepancy between the experiments and the CFD model in Fig. 12(a), since the mixture non-ideality can strongly affect the evaporation rate. In order to verify this hypothesis and overcome the lack of knowledge concerning the $\gamma_{i}\left(x_{i}\right)$ model, a fitting of the experimental data from Schmid et al. [37] has been performed using the Wilson model [40] for the activity coefficients $\gamma_{i}$ of the binary mixture:

$\ln \gamma_{1}=-\ln \left(x_{1}+x_{2} \Lambda_{1,2}\right)+x_{2}\left(\frac{\Lambda_{1,2}}{x_{1}+x_{2} \Lambda_{1,2}}-\frac{\Lambda_{2,1}}{x_{2}+x_{1} \Lambda_{2,1}}\right)$

$\ln \gamma_{2}=-\ln \left(x_{2}+x_{1} \Lambda_{2,1}\right)-x_{1}\left(\frac{\Lambda_{1,2}}{x_{1}+x_{2} \Lambda_{1,2}}-\frac{\Lambda_{2,1}}{x_{2}+x_{1} \Lambda_{2,1}}\right)$

where:

$\Lambda_{i, j}=\frac{v_{j}}{v_{i}} e^{-\frac{A_{i, j}}{R T}}$

in which $v$ represents the mole volume of the pure species. The two constants $A_{i, j}\left(A_{1,2}\right.$ and $\left.A_{2,1}\right)$ are the tuning parameters of the fitting procedure. The boiling pressure of the mixture can be easily calculated as a function of the acetic acid mole fraction $x_{1}$ :

$p\left(x_{1}\right)=p_{2}^{0} \gamma_{2}+x_{1}\left(p_{1}^{0} \gamma_{1}-p_{2}^{0} \gamma_{2}\right)$

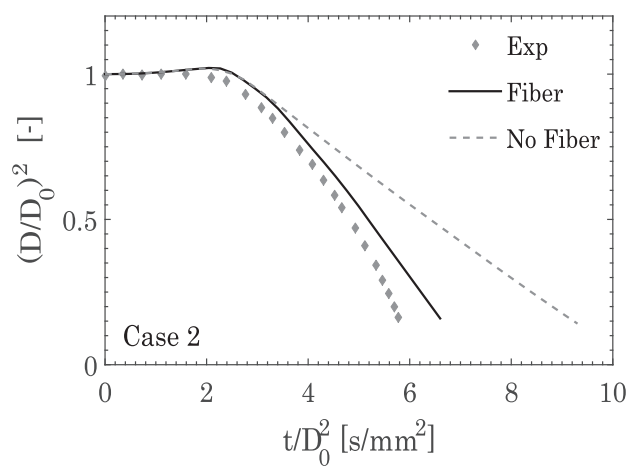

(a)

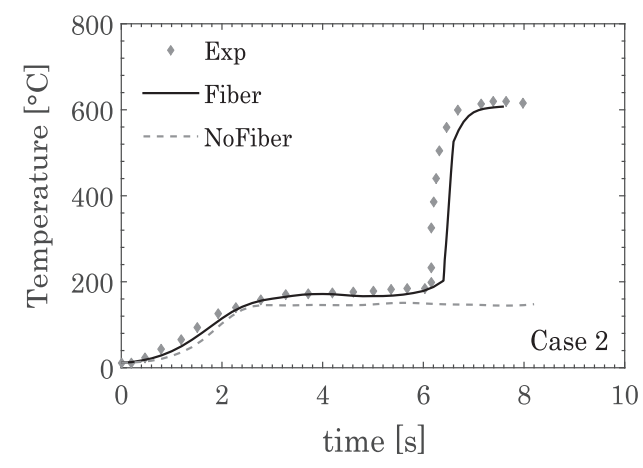

(b)

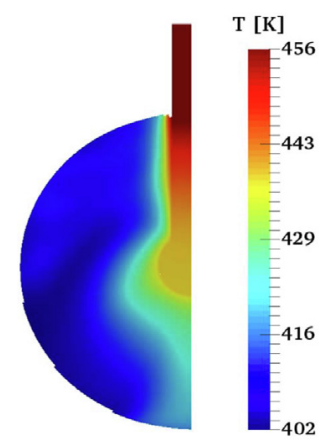

(c)

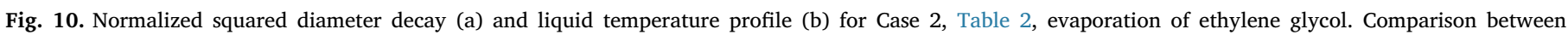

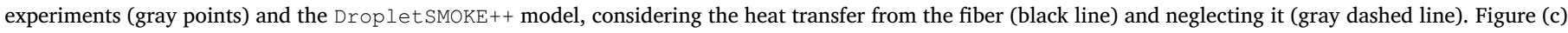
reports the temperature distribution along the solid fiber and inside the liquid droplet at time $\mathrm{t}=5 \mathrm{~s}$. 

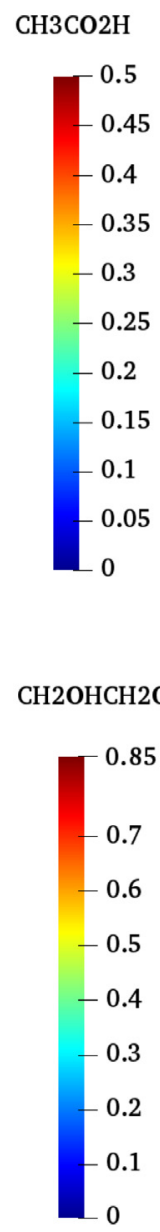

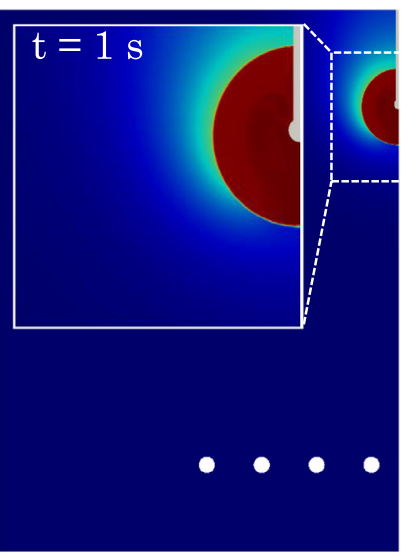

(a)

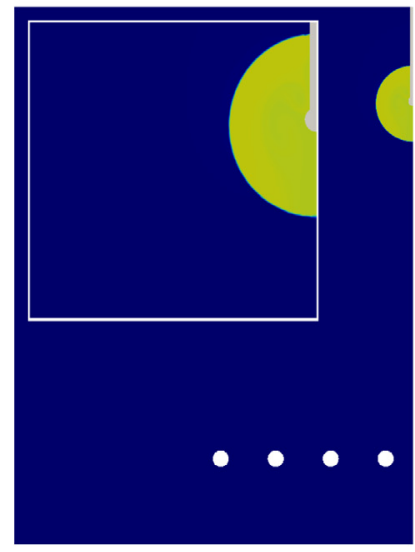

(d)

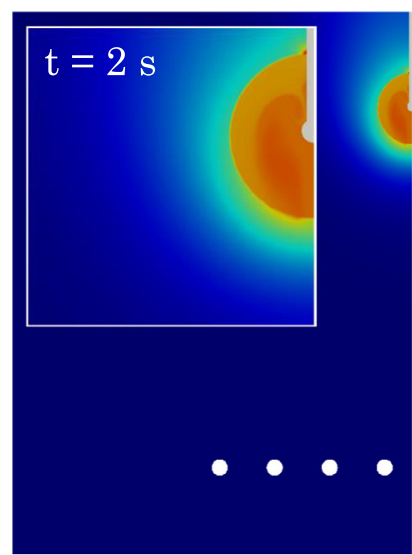

(b)

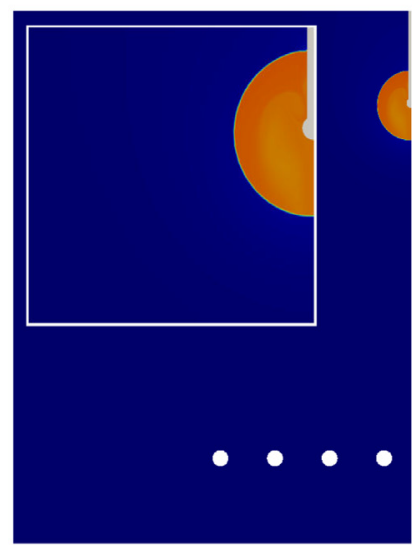

(e)

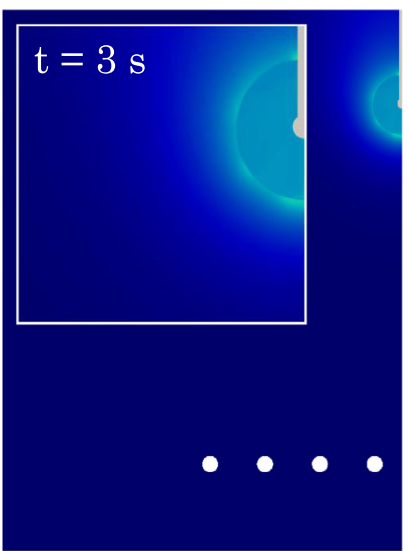

(c)

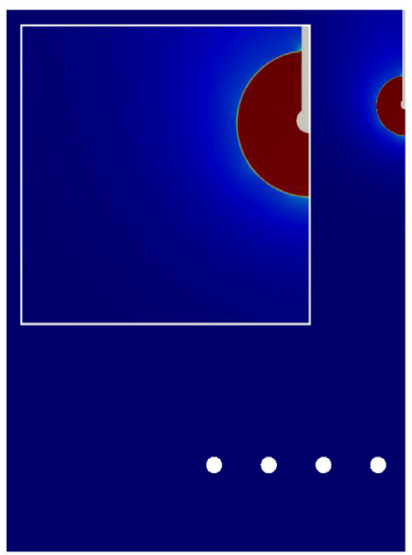

(f)

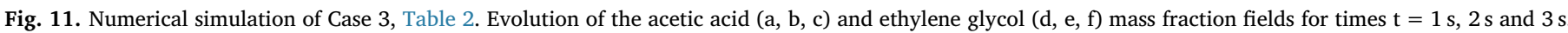

where $p_{i}^{0}$ is the vapor pressure of species $i$ at $T=363 \mathrm{~K}$. The two parameters $A_{1,2}$ and $A_{2,1}$ must be tuned in order to provide a boiling curve (Eq. (21)) which fits the experiments in Fig. 13(a). We obtain:

$\Lambda_{1,2}=0.977 e^{\frac{1920.09}{R T}}$

$\Lambda_{2,1}=1.022 e^{\frac{1545.9}{R T}}$

which inserted in Eqs. (18) and (19) provide the activity coefficients of

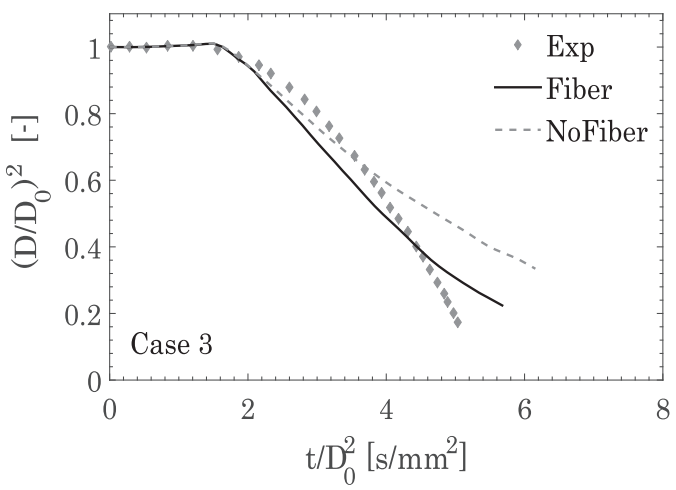

(a) acetic acid and ethylene glycol in function of local composition and temperature. The $\gamma_{i}$ curves are reported in Fig. 13(b) at $300 \mathrm{~K}$ (ambient $\mathrm{T}$ ) and $450 \mathrm{~K}$ (maximum $\mathrm{T}$ reached during the evaporation, Fig. 12b). It is clear that the values of $\gamma_{i}$ are far from ideality, especially within our domain of acetic acid concentration (from $\sim 0.5$ to 0 , Fig. 11). This model works in the direction we need, since we expect the acetic acid evaporation to be slightly delayed at the initial stages (since $\gamma_{\text {ac.acid }} \sim 0.65-0.7$ ), increasing the vaporization rate of ethylene glycol

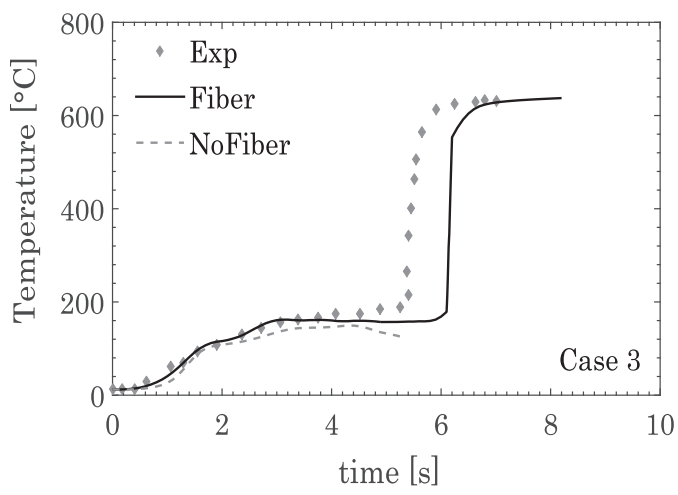

(b)

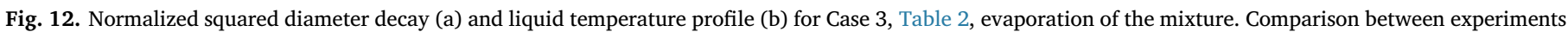

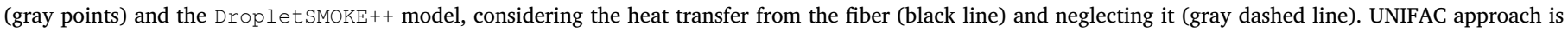
used for $\gamma_{i}$. 


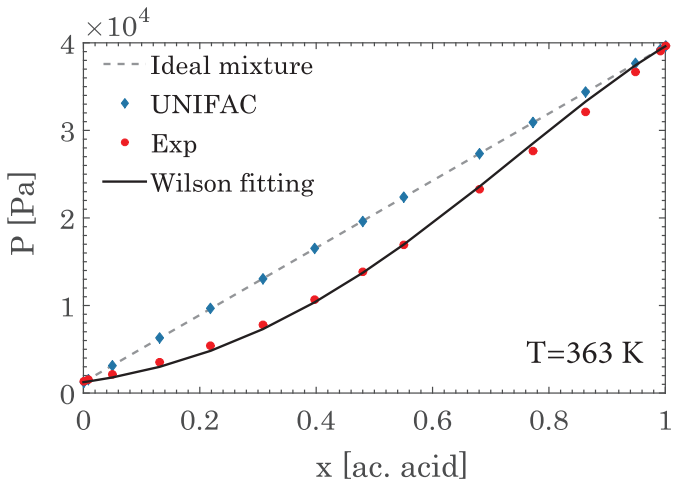

(a)

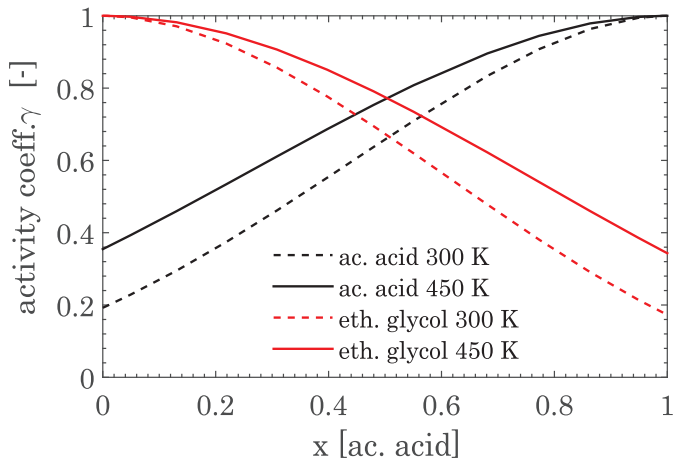

(b)

Fig. 13. VLE behavior (at $363 \mathrm{~K}$ ) of acetic acid-ethylene glycol mixtures (a) assuming ideal mixture and UNIFAC model for $\gamma_{i}$. The experimental data are taken from Schmid et al. [37] and the fitting is based on Wilson model. Figure (b) reports $\gamma_{i}$ at $300 \mathrm{~K}$ and $450 \mathrm{~K}$ using the Wilson fitted model.

towards the end (where $\gamma_{\text {et.glycol }}$ tends to 1 ).

Fig. 14 reports the result obtained with the Wilson model for the activity coefficients, showing a significant improvement of the model prediction. The concavity of the squared diameter profile (Fig. 12a) is not present anymore, and the diameter decay is almost linear with time. As for the pure components cases, the final stages of the evaporation present a slight delay with respect to the experiments. The fiber heats the liquid from the inside 14 (c) and provides the conditions for possible bubbles nucleation which would enhance the vaporization rate. It is interesting to notice that the steady-state temperature (plateau) reached by the droplet is very similar to the ethylene glycol droplet case (Fig. 10), even though the initial mixture is 1:1. Fig. 11(c) and (f) clearly show that at the time when the plateau is reached (around 3-4 s), the mixture composition is almost totally shifted toward ethylene glycol. The more volatile component (acetic acid) is almost completely evaporated.

\subsection{Effect of the fiber on the droplet heating}

It is clear from the previous results that the solid fiber can have a significant effect on the vaporization phenomena. It is interesting to quantitatively investigate the contribution of the fiber to the global heat flux on the droplet. To this purpose we performed a post-processing analysis on the heat fluxes on the liquid droplet, divided in:

- Heat flux on the droplet surface $S$

$$
\dot{Q}_{c o n v}=\int_{S}\left(k \nabla T+\rho C_{p} T \vec{v}\right) \vec{n} d S
$$

- Evaporation flux

$$
\dot{Q}_{e v}=\int_{S} \sum_{i}^{N_{S}} \dot{m}_{i} \Delta h_{e v, i} d S
$$

- Conductive flux from the fiber surface $S_{f}$

$$
\dot{Q}_{f i b}=\int_{S_{f}} k_{s} \nabla T_{s} \vec{n} d S_{f}
$$

The results are presented in Fig. 15(a) for the pure acetic acid droplet evaporation. The upward convective flow heats the thermocouple faster than the droplet, because of the initial surface cooling due to vaporization. This makes the fiber contribution significantly important at the initial stages of the evaporation process. Later on, the fiber contribution is stabilized at an almost constant value (due to the reaching of a constant droplet temperature) and has a sudden increase towards the end of the vaporization due to the complete droplet evaporation. This can be seen more in detail in Fig. 15(b), where the $\dot{Q}_{f i b}$ contribution is highlighted through the ratio $\frac{\dot{Q}_{f i}}{\dot{Q}_{i n}}=\frac{\dot{Q}_{f i b}}{\dot{Q}_{c o n v}+\dot{Q}_{f i b}}$, representing the relative contribution of the heat conduction from the fiber to the total heat flux income. The peak of the $\dot{Q}_{f i b}$ contribution can reach $30-40 \%$ of the total heat flux income at the very beginning of the vaporization (when the difference between the liquid and the fiber temperature is maximum), going down to $8-10 \%$ throughout the process. This is however sufficient to provide a $10-15 \mathrm{~K}$ increase in the

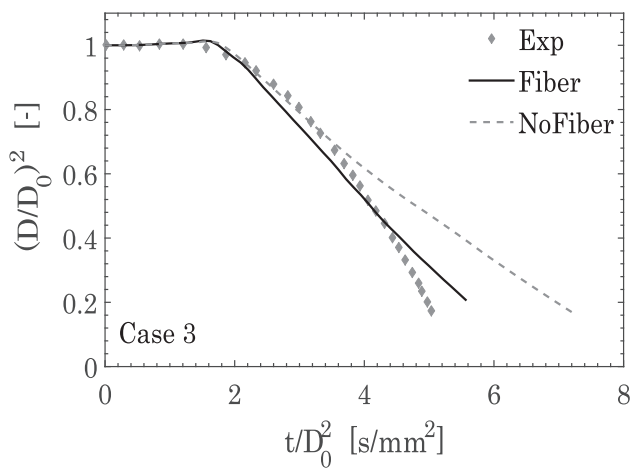

(a)

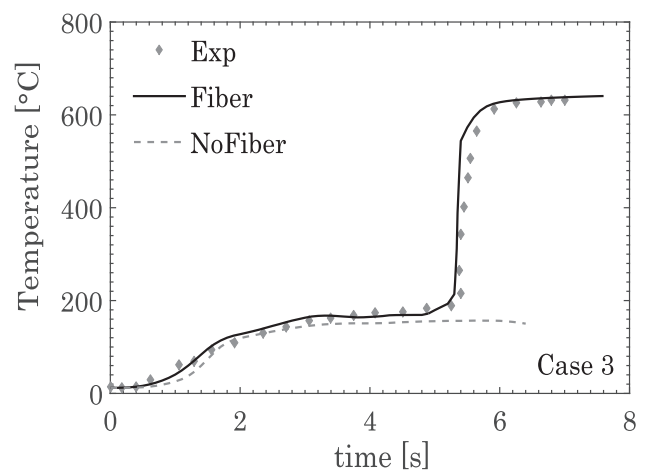

(b)

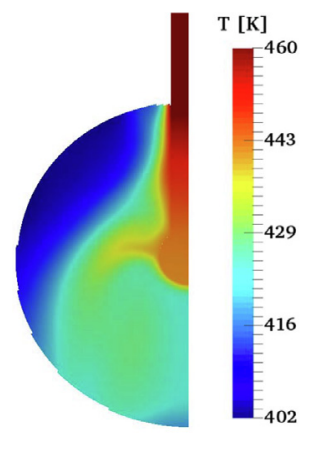

(c)

Fig. 14. Normalized squared diameter decay (a) and liquid temperature profile (b) for Case 3, Table 2, evaporation of the mixture. Comparison between experiments (gray points) and the DropletSMOKE++ model, considering the heat transfer from the fiber (black line) and neglecting it (gray dashed line). Wilson model used for $\gamma_{i}$. Figure (c) reports the temperature distribution along the solid fiber and inside the liquid droplet at time $t=2 \mathrm{~s}$. 


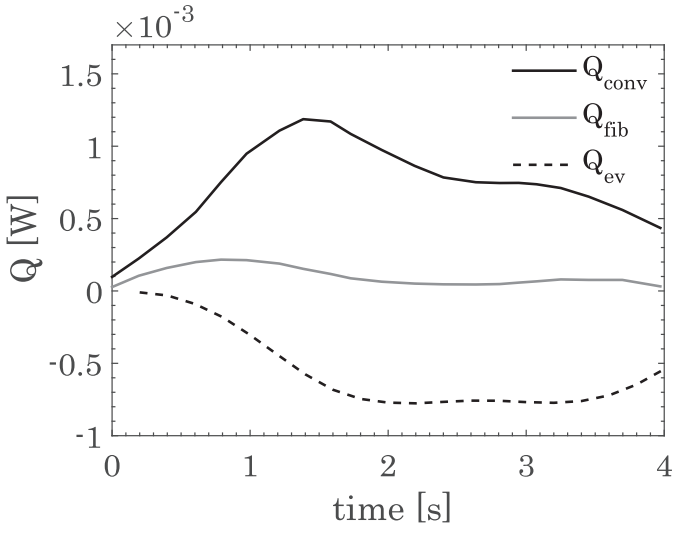

(a)

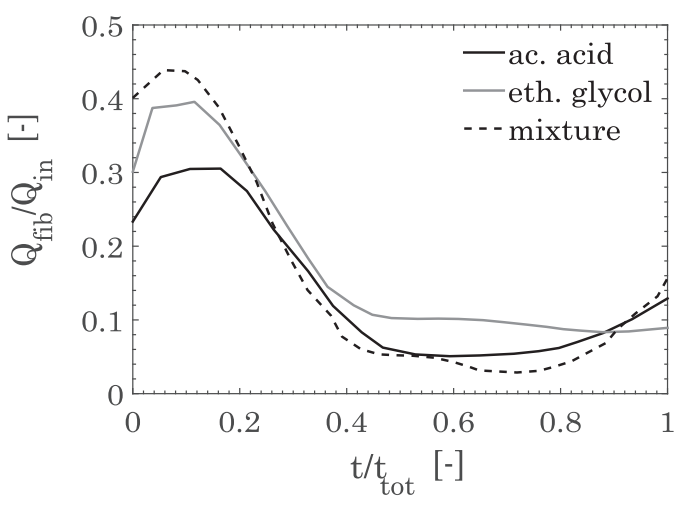

(b)

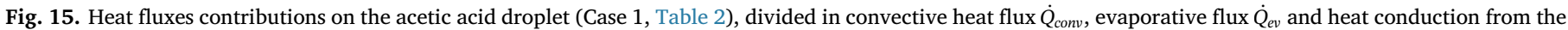
fiber $\dot{Q}_{f i b}$ (a). Relative contribution of $\dot{Q}_{f i b}$ to the total income $Q_{i n}=\dot{Q}_{c o n v}+\dot{Q}_{f i b}$ (b) for the three cases in Table 2 .

droplet temperature (Fig. 9 and a consequent reduced droplet lifetime. These results are in agreement with a few studies available in literature on the same topic. Yang et al. [16] investigated with a simple onedimensional model the effect of supporting fibers on n-heptane and n-hexadecane droplet evaporation, showing the same qualitative trend we presented in our analysis. The importance of $\dot{Q}_{f i b}$ at the early stage of the evaporation has also been described by Han et al. [18] and Harada et al. [31] for different support fibers, describing a situation in which the solid fiber has been sufficiently heated by the hot gases, while the droplet temperature is still low.

\subsection{Internal circulation and preferential vaporization}

The presence of internal convection in the droplet is a well known phenomenon from the experimental point of view. By means of Planar Laser-Induced Fluorescence and Particle Image Velocimetry [41,42] and laser scattering $[43,44]$ it is possible to get an insight on the internal motion in the liquid phase.

Since we are solving for the whole velocity field, the liquid convection can also be visualized numerically. The internal velocity field for the acetic acid droplet evaporation are reported in Fig. 16 for three different times. The main mechanism which leads to the formation of vortices in the liquid phase is the shear force applied on the interface by the external convection, as reported by Sirignano [2]. This can generate an internal flow field which can be of the order of $5-20 \%$ of the free stream velocity [45]. Density gradients due to temperature differences inside the droplet usually have a secondary effect. The shear force induces the formation of a main vortex close to the droplet surface. This latter vortex, to enforce flow continuity, induces a second smaller vortex which rotates in opposite sense (Fig. 16). The dimension of the main vortex is reduced during evaporation and its center is shifted toward the center of the droplet. This also causes a reduction of the second vortex and eventually its extinction (Fig. $16 \mathrm{c}$ ). This is in agreement to what reported by the experimental work of Volkov et al. [41] on water droplets evaporation, where they detect a lower intensity of the internal convection as the evaporation proceeds.

It is important to point out that internal convection can have a major impact on the evaporation rate, especially concerning multicomponent droplets. If the volatilities of the components are very different (such as in real fuels), preferential vaporization can take place. This phenomenon is strongly dependent on the liquid flow field $[46,45]$ : if liquid convection is weak the droplet will most likely evaporate through "layers" (shell model), making internal diffusion the limiting step of the evaporation process. On the other hand, if internal convection is strong enough, the concentration of the components would be almost uniform in the liquid phase. The more volatile components are continuously transported toward the droplet surface, facilitating their preferential vaporization.

This can be clearly seen in Fig. 12(b) for the evaporation of the acetic acid-ethylene glycol mixture: before reaching the plateau, the

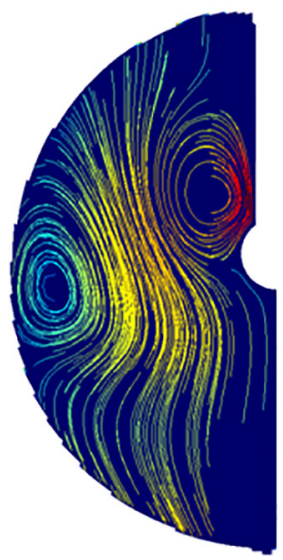

(a)

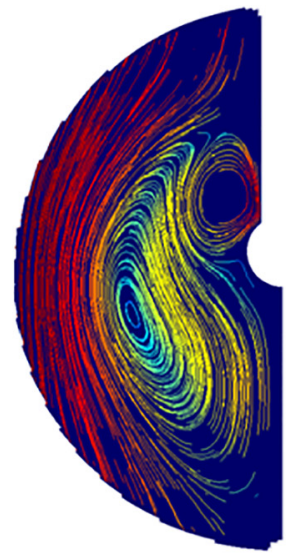

(b)
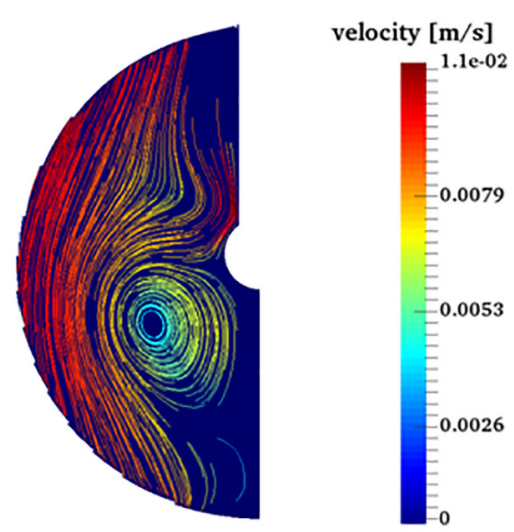

(c)

Fig. 16. Flow field streamlines in the liquid phase, acetic acid droplet evaporation (Case 1, Table 2). Times $t=0.4 \mathrm{~s}$ (a), $1.2 \mathrm{~s}$ (b), $2 \mathrm{~s}$ (c). 
droplet temperature is more irregular if compared to the pure monocomponent cases (Figs. 9 and 10(b)) showing two changes in the profile concavity. This is behavior is typical of multi-stage vaporization and it is also exhibited by the diameter decay profile (Fig. 12a) where it is evident a change in the profile slope (i.e. the vaporization rate). The internal convection (Fig. 16) plays a key role in the preferential vaporization of the more volatile component (acetic acid in this case) because it significantly accelerates the internal mass transfer, which would be the limiting step in case of pure internal diffusion (in the absence of internal mixing). Finally it is worth noticing that when more realistic activity coefficients $\gamma_{i}$ are accounted for (by the Wilson model, Fig. 14) the preferential vaporization is smoothed and less evident. This is due to the stronger molecular interactions between the two components, which modify their relative volatility (Fig. 13 a) and make the mixture evaporate similarly to monocomponent cases.

\section{Conclusions}

In this work we presented an experimental and numerical activity on the evaporation of suspended fuel droplets in a convective flow. Acetic acid and ethylene glycol (and their mixture) have been studied because of their importance as components of complex fuel surrogates. The droplets have been suspended on a support fiber in a small combustion chamber and evaporated in buoyancy driven convection, triggered by the electrical heating of a resistive coil placed under the droplet. The support fiber is a thermocouple, used to measure the heating rate and the liquid temperature of the droplet. The experiments have been then modeled with the multiphase CFD code DropletSMOKE ++ , based on the VOF methodology for the interface tracking. In this work the code was properly extended to account for the conjugate heat transfer between the solid support fiber and the fluid region (which includes both the liquid and the gas in the VOF description). The numerical results provide useful information on the flow field around and inside the droplet, as well as the gas, the liquid and the solid temperature distribution. The evaporation rate is shown to be largely underpredicted if the heat transfer from the support fiber is neglected (considering the boundary as adiabatic), both for acetic acid and ethylene glycol droplets. The multiregion approach effectively describes the additional heat flux conducted through the thermocouple, which clearly modifies the internal temperature distribution. The heat flux from the droplet center is advected inside the liquid phase and toward the surface, accelerating the vaporization and providing a good agreement with the experimental profiles. The DropletSMOKE++ prediction of the mixture evaporation has been significantly improved using the activity coefficients obtained from a fitting of the VLE experiments, showing the inability of the available methods (UNIFAC, ASOG) to model the acetic acid-ethylene glycol mixture thermodynamics. Finally, the numerical results allowed to investigate the internal convection in the liquid phase and its formation mechanism. The strong internal mixing allows to preferentially vaporize the more volatile component, leading to a double stage evaporation.

Future works will be focused on the extension of the evaporation model in order to consider the nucleation and boiling inside the liquid phase, as well as the introduction of a gas phase kinetics to simulate the combustion phenomenon.

\section{Acknowledgments}

The Residue2Heat project has received funding from the European Union's Horizon 2020 research and innovation programme under grant agreement No 654650 .

\section{References}

[1] B. Abramzon, W. Sirignano, Droplet vaporization model for spray combustion calculations, Int. J. Heat Mass Transf. 32 (9) (1989) 1605-1618.
[2] W.A. Sirignano, Fluid Dynamics and Transport of Droplets and Sprays, Cambridge University Press, 1999.

[3] H. Ghassemi, S.W. Baek, Q.S. Khan, Experimental study on binary droplet evaporation at elevated pressures and temperatures, Combust. Sci. Technol. 178 (6) (2006) 1031-1053.

[4] K. Han, C. Zhao, G. Fu, F. Zhang, S. Pang, Y. Li, Evaporation characteristics of dual component droplet of benzyl azides-hexadecane mixtures at elevated temperatures, Fuel 157 (2015) 270-278.

[5] S. Sazhin, M. Al Qubeissi, R. Kolodnytska, A. Elwardany, R. Nasiri, M. Heikal, Modelling of biodiesel fuel droplet heating and evaporation, Fuel 115 (2014) 559-572.

[6] S.S. Sazhin, Advanced models of fuel droplet heating and evaporation, Progr. Energy Combust. Sci. 32 (2) (2006) 162-214.

[7] A.J. Marchese, F.L. Dryer, V. Nayagam, Numerical modeling of isolated n-alkane droplet flames: initial comparisons with ground and space-based microgravity experiments, Combust. Flame 116 (3) (1999) 432-459.

[8] J. Piskorz, D. Scott, D. Radlein, Pyrolysis oils from biomass: Producing analyzing and upgrading, Symposium Series, vol. 376, American Chemical Society Washington, DC, 1988.

[9] A. Oasmaa, C. Peacocke, et al., A guide to physical property characterisation of biomass-derived fast pyrolysis liquids, Technical Research Centre of Finland Espoo (2001).

[10] A. Oasmaa, D. Meier, Norms and standards for fast pyrolysis liquids: 1. Round robin test, J. Anal. Appl. Pyrol. 73 (2) (2005) 323-334.

[11] Residue2Heat,https://www.residue2heat.eu/.

[12] R. Calabria, F. Chiariello, P. Massoli, Combustion fundamentals of pyrolysis oil based fuels, Exp. Thermal Fluid Sci. 31 (5) (2007) 413-420.

[13] M. Pelucchi, C. Cavallotti, A. Cuoci, T. Faravelli, A. Frassoldati, E. Ranzi, Detailed kinetics of substituted phenolic species in pyrolysis bio-oils, React. Chem. Eng.

[14] A. Saufi, A. Frassoldati, T. Faravelli, A. Cuoci, DropletSMOKE + + : a comprehensive multiphase CFD framework for the evaporation of multidimensional fuel droplets, Int. J. Heat Mass Transf. 131 (2019) 836-853.

[15] J.-R. Yang, S.-C. Wong, On the discrepancies between theoretical and experimental results for microgravity droplet evaporation, Int. J. Heat Mass Transf. 44 (23) (2001) 4433-4443.

[16] J.-R. Yang, S.-C. Wong, An experimental and theoretical study of the effects of heat conduction through the support fiber on the evaporation of a droplet in a weakly convective flow, Int. J. Heat Mass Transf. 45 (23) (2002) 4589-4598.

[17] H.L.-U. Rehman, J. Weiss, P. Seers, Effect of heat conduction on droplet life time and evaporation rate under forced convection at low temperatures, Exp. Thermal Fluid Sci. 72 (2016) 59-66.

[18] K. Han, G. Song, X. Ma, B. Yang, An experimental and theoretical study of the effect of suspended thermocouple on the single droplet evaporation, Appl. Therm. Eng. 101 (2016) 568-575.

[19] D. Shringi, H. Dwyer, B. Shaw, Influences of support fibers on vaporizing fuel droplets, Comput. Fluids 77 (2013) 66-75.

[20] N. Ghata, B.D. Shaw, Computational modeling of the effects of support fibers on evaporation of fiber-supported droplets in reduced gravity, Int. J. Heat Mass Transf. 77 (2014) 22-36.

[21] P. Strizhak, R. Volkov, G. Castanet, F. Lemoine, O. Rybdylova, S. Sazhin, Heating and evaporation of suspended water droplets: experimental studies and modelling, Int. J. Heat Mass Transf. 127 (2018) 92-106.

[22] C.W. Hirt, B.D. Nichols, Volume of fluid (VOF) method for the dynamics of free boundaries, J. Comput. Phys. 39 (1) (1981) 201-225.

[23] G. Tryggvason, R. Scardovelli, S. Zaleski, Direct Numerical Simulations of Gas-liquid Multiphase Flows, Cambridge University Press, 2011.

[24] A.P. Pinheiro, J.M. Vedovoto, Evaluation of droplet evaporation models and the incorporation of natural convection effects, Flow Turbul. Combust. 1-22.

[25] J.M. Smith, Introduction to Chemical Engineering Thermodynamics, ACS Publications, 1950.

[26] A. Fredenslund, R.L. Jones, J.M. Prausnitz, Group-contribution estimation of activity coefficients in nonideal liquid mixtures, AIChE J. 21 (6) (1975) 1086-1099.

[27] J. Leffler, H.T. Cullinan, Jr, Variation of liquid diffusion coefficients with composition. Dilute ternary systems, Ind. Eng. Chem. Fundam. 9 (1) (1970) 88-93.

[28] J. Brackbill, D.B. Kothe, C. Zemach, A continuum method for modeling surface tension, J. Comput. Phys. 100 (2) (1992) 335-354.

[29] S. Popinet, Numerical models of surface tension, Annu. Rev. Fluid Mech. 50 (2018) 49-75.

[30] A. Albadawi, D. Donoghue, A. Robinson, D. Murray, Y. Delauré, Influence of surface tension implementation in volume of fluid and coupled volume of fluid with level set methods for bubble growth and detachment, Int. J. Multiph. Flow 53 (2013) $11-28$.

[31] T. Harada, H. Watanabe, Y. Suzuki, H. Kamata, Y. Matsushita, H. Aoki, T. Miura, A numerical investigation of evaporation characteristics of a fuel droplet suspended from a thermocouple, Int. J. Heat Mass Transf. 54 (1-3) (2011) 649-655.

[32] C. Chauveau, M. Birouk, F. Halter, I. Gökalp, An analysis of the droplet support fiber effect on the evaporation process, Int. J. Heat Mass Transf. 128 (2019) 885-891.

[33] A. Cuoci, A. Frassoldati, T. Faravelli, E. Ranzi, OpenSMOKE + +: an object-oriented framework for the numerical modeling of reactive systems with detailed kinetic mechanisms, Comput. Phys. Commun. 192 (2015) 237-264.

[34] C.L. Yaws, The yaws handbook of physical properties for hydrocarbons and chemicals: physical properties for more than 54,000 organic and inorganic chemical compounds, Coverage for C1 to C100 Organics and Ac to Zr Inorganics, Professional Publishing, Gulf, 2015.

[35] R.C. Reid, J.M. Prausnitz, B.E. Poling, The properties of gases and liquids.

[36] C.J. Greenshields, OpenFOAM user guide, OpenFOAM Foundation Ltd, version 
$3(1)$.

[37] B. Schmid, M. Döker, J. Gmehling, Measurement of the thermodynamic properties for the reactive system ethylene glycol-acetic acid, Fluid Phase Equilib. 258 (2) (2007) 115-124.

[38] J. Lohmann, R. Joh, J. Gmehling, From UNIFAC to modified UNIFAC (Dortmund), Ind. Eng. Chem. Res. 40 (3) (2001) 957-964.

[39] A. Rizzi, J.F. Huber, Comparative calculations of activity coefficients in binary liquid mixtures at infinite dilution using the solution of groups model, Ind. Eng. Chem. Process Des. Develop. 20 (2) (1981) 204-210.

[40] F.A. Gothard, M.F.C. Ciobanu, D.G. Breban, C.I. Bucur, G.V. Sorescu, Predicting the parameters in the Wilson equations for activity coefficients in binary hydrocarbon systems, Ind. Eng. Chem. Process Des. Develop. 15 (2) (1976) 333-337.

[41] R. Volkov, P. Strizhak, Research of temperature fields and convection velocities in evaporating water droplets using planar laser-induced fluorescence and particle image velocimetry, Exp. Thermal Fluid Sci. 97 (2018) 392-407.

[42] R. Volkov, P. Strizhak, Measuring the temperature of a rapidly evaporating water droplet by planar laser induced fluorescence, Measurement 135 (2019) 231-243.

[43] D.K. Mandal, S. Bakshi, Evidence of oscillatory convection inside an evaporating multicomponent droplet in a closed chamber, J. Colloid Interface Sci. 378 (1) (2012) 260-262.

[44] D.K. Mandal, S. Bakshi, Internal circulation in a single droplet evaporating in a closed chamber, Int. J. Multiph. Flow 42 (2012) 42-51.

[45] C.K. Law, S. Prakash, W. Sirignano, Theory of convective, transient, multicomponent droplet vaporization, Symposium (International) on Combustion, vol. 16, Elsevier, 1977, pp. 605-617.

[46] C.K. Law, Multicomponent droplet combustion with rapid internal mixing, Combust. Flame 26 (1976) 219-233. 